Supporting Information Binder, Kirsch

\title{
Synthesis of Highly Substituted Pyrroles via a Multimetal- Catalyzed Rearrangement-Condensation-Cyclization Domino Approach
}

\author{
Jörg T. Binder, Stefan F. Kirsch* \\ Department Chemie, Technische Universität München, Lichtenbergstr. 4, D-85747 Garching, \\ Germany
}

\section{Supporting Information}

Representative experimental procedures for catalytic pyrrole formation, and copies of ${ }^{1} \mathrm{H}$ and ${ }^{13} \mathrm{C}$ NMR of 3 and 5.

(35 Pages)

\section{List of Contents}

$\mathrm{S} 1-\mathrm{S} 2$

General experimental details

S2

General procedure for the pyrrole formation starting from propargyl vinyl ethers 1

S2-S9 Further characterization data for compounds $\mathbf{3}$

S10-S31

${ }^{1} \mathrm{H}$ NMR and ${ }^{13} \mathrm{C}$ NMR spectra for compounds 3

S32-S35

${ }^{1} \mathrm{H}$ NMR and ${ }^{13} \mathrm{C}$ NMR spectra for compounds 5

General experimental details: All propargyl vinyl ethers $\mathbf{1}$ were synthesized by the reaction of the corresponding propargylic alcohols with 2-propynoic acid derivatives in the presence of trimethylphosphine or tributylphosphine. ${ }^{1}$ 3-Butin-1-ol was purchased from Acros; and ethyl 2butynoate, methyl 2-octynoate, ethyl phenylpropiolate and 3-pentyn-2-ol were purchased from Aldrich. All commercially available chemicals were used without further purification. All the other propargylic alcohols were prepared from the corresponding alkynes by addition to paraformaldehyde. ${ }^{2}$ $\mathrm{AgSbF}_{6}$ and $\left(\mathrm{Ph}_{3} \mathrm{P}\right) \mathrm{AuCl}$ were purchased from Aldrich.

\footnotetext{
${ }^{1}$ Inanaga, J.; Baba, Y.; Hanamoto, T. Chem. Lett. 1993, 241-244.

${ }^{2}$ Hashmi, A. S. K.; Haufe, P.; Nass, A. R. Adv. Synth. Catal. 2003, 345, 1237-1241.
} 
${ }^{1} \mathrm{H}$ NMR spectra were obtained on Bruker $500 \mathrm{MHz}$ FT-NMR, $360 \mathrm{MHz}$ FT-NMR and $250 \mathrm{MHz}$ FTNMR spectrometers. ${ }^{13} \mathrm{C}$ NMR spectra were recorded at $90.6 \mathrm{MHz}$. Chemical shifts are reported in ppm relative to solvent signal. Multiplicity is indicated as follows: s (singlet); d (doublet); t (triplet); q (quartet); m (multiplet); dd (doublet of doublets); sep (septet). High resolution mass spectra and EI were determined on a Finnigan MAT 95S and MAT 8200. Flash chromatography was performed with neutral $\mathrm{Al}_{2} \mathrm{O}_{3}$ (Aldrich; Brockmann 1). ${ }^{3}$ The eluent used is reported in parentheses ( $\mathrm{P}=$ pentanes). Thin-layer chromatography (TLC) was performed on precoated glass-backed plates (Merck Kieselgel $60 \mathrm{~F}_{254}$ ), and components were visualized by observation under UV light or by treating the plates with $\mathrm{KMnO}_{4} / \mathrm{H}_{2} \mathrm{SO}_{4}$ followed by heating.

\section{General Procedure for the Pyrrole Formation starting from Propargyl}

\section{Vinyl Ethers 1.}

\section{Ethyl 1-(3-chlorophenyl)-4,5-dimethyl-2-phenylpyrrole-3-carboxylate (3af)}

$\mathrm{AgSbF}_{6}$ (3.4 mg, $5 \mathrm{~mol} \%$ ) was added to a solution of $1 \mathrm{a}$ (50 mg, $\left.0.20 \mathrm{mmol}\right)$ in $\mathrm{CH}_{2} \mathrm{Cl}_{2}(0.5$ $\mathrm{ml}$ ), and the reaction vial was sealed protected from light, and stirred at room temperature for $10 \mathrm{~min}$. Then, 3-chloro-aniline (39.2 mg, $0.31 \mathrm{mmol}, 1.5 \mathrm{eq})$ and $\left(\mathrm{Ph}_{3} \mathrm{P}\right) \mathrm{AuCl}(5.1 \mathrm{mg}, 5$ mol\%) were added subsequently. The dark reaction mixture was stirred at $38^{\circ} \mathrm{C}$ for $1 \mathrm{~h}$ (until TLC analysis indicated complete conversion). The mixture was concentrated under reduced pressure. Purification of the residue by flash chromatography on neutral $\mathrm{Al}_{2} \mathrm{O}_{3}(\mathrm{P} / \mathrm{EtOAc}=$ 98/2) gave pyrrole 3af as a colorless solid (59.0 mg, $0.17 \mathrm{mmol}, 83 \%) . \mathrm{R}_{\mathrm{f}}=0.73(\mathrm{P} / \mathrm{EtOAc}=$ 80/20); ${ }^{1} \mathrm{H}$ NMR (360 MHz, $\mathrm{CDCl}_{3}$ ): $\delta=1.04$ (t, $\left.J=7.2 \mathrm{~Hz}, 3 \mathrm{H}\right), 2.01$ (s, $3 \mathrm{H}$ ), 2.31 (s, $3 \mathrm{H}$ ), 4.08 (q, $J=7.2 \mathrm{~Hz}, 2 \mathrm{H}), 6.88-6.90$ (m, $1 \mathrm{H}), 7.05-7.06$ (m, $1 \mathrm{H}), 7.09-7.12(\mathrm{~m}, 2 \mathrm{H})$, 7.15-7.24 (m, $5 \mathrm{H}) ;{ }^{13} \mathrm{C}$ NMR (90.6 MHz, $\left.\mathrm{CDCl}_{3}\right): \delta=10.7,11.1,14.1$, 59.4, 113.4, 117.2, 127.1, 127.2, 127.5, 127.6, 128.2, 129.1, 129.8, 131.2, 132.5, 134.4, 137.9, 139.6, 165.8; IR (cm $\left.{ }^{-1}\right): 2924$ (m), 1700 (vs), 1592 (m), 1481 (s), 1380 (m), 1259 (m), 1147 (s), 1069 (m);

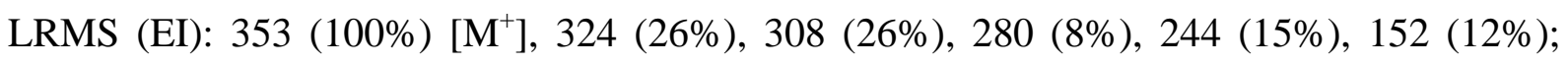
HRMS 353.1182 [353.1183 calcd for $\left.\mathrm{C}_{21} \mathrm{H}_{20} \mathrm{NO}_{2} \mathrm{Cl}\left(\mathrm{M}^{+}\right)\right]$.

\footnotetext{
${ }^{3}$ The use of neutral $\mathrm{Al}_{2} \mathrm{O}_{3}$ for column chromatography was neccessary to ensure reproducible yields.
} 


\section{Ethyl 4,5-dimethyl-2-phenyl-1-phenylpyrrole-3-carboxylate (3aa)}

Following the general procedure, 3aa was obtained as a colorless solid (71\%) after flash chromatography on neutral $\mathrm{Al}_{2} \mathrm{O}_{3}(\mathrm{P} /$ EtOAc $=95 / 5) . \mathrm{R}_{\mathrm{f}}=0.73(\mathrm{P} / \mathrm{EtOAc}=80 / 20) ;{ }^{1} \mathrm{H} \mathrm{NMR}$ (360 MHz, $\left.\mathrm{CDCl}_{3}\right): \delta=1.07$ (t, $J=7.2 \mathrm{~Hz}, 3 \mathrm{H}$ ), 2.02 (s, $3 \mathrm{H}$ ), 2.02 (s, $3 \mathrm{H}$ ), 4.11 (q, $J=7.2$ Hz, 2 H), 7.02-7.05 (m, 2 H), 7.11-7.17 (m, 5 H), 7.22-7.25 (m, 1 H), 7.28-7.30 (m, 2 H); ${ }^{13} \mathrm{C}$ NMR (90.6 MHz, $\left.\mathrm{CDCl}_{3}\right): \delta=10.7,11.2,14.1,59.3,113.0,116.8,127.3,127.3,127.3$, 127.3, 127.8, 128.8, 131.2, 132.9, 137.9, 138.4, 165.9; IR (cm-1): 2926 (m), 1698 (s), 1498 (m), 1269 (s), 1179 (s), 1080 (m), 763 (m), 697 (s); LRMS (EI): 319 (100\%) [M'], 290 (26\%), 274 (29\%), 258 (10\%), 244 (13\%), 230 (12\%), 128 (13\%), 118 (18\%), 105 (40\%), 77 (30\%), 71 (17\%), 57 (27\%), 43 (28\%); HRMS 319.1573 [319.1572 calcd for $\mathrm{C}_{21} \mathrm{H}_{21} \mathrm{NO}_{2}$ $\left.\left(\mathrm{M}^{+}\right)\right]$.

\section{Ethyl 4,5-dimethyl-2-phenyl-1-(4-methoxyphenyl)-pyrrole-3-carboxylate (3ab)}

Following the general procedure, 3ab was obtained as a colorless solid (75\%) after flash chromatography on neutral $\mathrm{Al}_{2} \mathrm{O}_{3}(\mathrm{P} /$ EtOAc $=95 / 5) . \mathrm{R}_{\mathrm{f}}=0.58(\mathrm{P} / \mathrm{EtOAc}=80 / 20) ;{ }^{1} \mathrm{H} \mathrm{NMR}$ (360 MHz, $\left.\mathrm{CDCl}_{3}\right): \delta=1.04$ (t, $\left.J=7.0 \mathrm{~Hz}, 3 \mathrm{H}\right), 1.98$ (s, $\left.3 \mathrm{H}\right), 2.31$ (s, $\left.3 \mathrm{H}\right), 3.76(\mathrm{~s}, 3 \mathrm{H})$, 4.08 (q, $J=7.0 \mathrm{~Hz}, 2 \mathrm{H}), 6.75-6.78$ (m, $2 \mathrm{H}), 6.91-6.94$ (m, $2 \mathrm{H}), 7.10-7.16(\mathrm{~m}, 5 \mathrm{H}) ;{ }^{13} \mathrm{C}$ NMR (90.6 MHz, $\left.\mathrm{CDCl}_{3}\right): \delta=10.7,11.2,14.1,55.5,59.2,112.8,114.0,116.6,127.2,127.3$, 127.6, 129.8, 131.2, 131.2, 133.0, 138.2, 158.9, 166.0; IR (cm $\left.{ }^{-1}\right)$ : 2924 (s), 1693 (s), 1607 (w), 1514 (s), 1480 (m), 1386 (m), 1248 (s), 1148 (m), 1068 (m), 1036 (m), 837 (m); LRMS (EI): 349 (100\%) [ $\left.\mathrm{M}^{+}\right], 320$ (20\%), 304 (20\%), 276 (10\%), 105 (22\%); HRMS 349.1675 [349.1678 calcd for $\left.\mathrm{C}_{22} \mathrm{H}_{23} \mathrm{NO}_{3}\left(\mathrm{M}^{+}\right)\right]$.

\section{Ethyl 4,5-dimethyl-2-phenyl-1-(4-iso-propylphenyl)-pyrrole-3-carboxylate (3ac)}

Following the general procedure, 3ac was obtained as a brown solid (73\%) after flash chromatography on neutral $\mathrm{Al}_{2} \mathrm{O}_{3}(\mathrm{P} /$ EtOAc $=95 / 5) . \mathrm{R}_{\mathrm{f}}=0.80(\mathrm{P} /$ EtOAc $=80 / 20) ;{ }^{1} \mathrm{H} \mathrm{NMR}$ (360 MHz, $\mathrm{CDCl}_{3}$ ): $\delta=1.05$ (t, $J=7.2 \mathrm{~Hz}, 3 \mathrm{H}$ ), 1.20 (d, $J=6.8 \mathrm{~Hz}, 6 \mathrm{H}$ ), 2.00 (s, $3 \mathrm{H}$ ), 2.32 (s, 3 H), 2.85 (sep, $J=6.8$ Hz, 1 H), 4.09 (q, $J=7.2$ Hz, 2 H), 6.90-6.93 (m, 2 H), 7.08-7.13 (m, $7 \mathrm{H}) ;{ }^{13} \mathrm{C}$ NMR (90.6 MHz, $\left.\mathrm{CDCl}_{3}\right): \delta=10.8,11.2,14.1,23.9,33.8,59.2,112.8,116.7$, 126.7, 127.2, 127.2, 127.5, 128.5, 131.2, 133.0, 135.9, 137.9, 148.5, 166.0; IR (cm $\left.{ }^{-1}\right): 2925$ (m), 1698 (s), 1515 (m), 1264 (m), 1030 (s), 740 (m); LRMS (EI): 361 (96\%) [M+], 332 (16\%), 316 (18\%), 199 (14\%), 105 (100\%); HRMS 361.2041 [361.2042 calcd for $\mathrm{C}_{24} \mathrm{H}_{27} \mathrm{NO}_{2}$ $\left.\left(\mathrm{M}^{+}\right)\right]$. 


\section{Ethyl 4,5-dimethyl-2-phenyl-1-(4-hydroxyphenyl)-pyrrole-3-carboxylate (3ad)}

Following the general procedure, 3ad was obtained as a yellow solid (41\%) after flash chromatography on silica. $(\mathrm{P} / \mathrm{EtOAc}=90 / 10) . \mathrm{R}_{\mathrm{f}}=0.25(\mathrm{P} / \mathrm{EtOAc}=80 / 20) ;{ }^{1} \mathrm{H}$ NMR $(360$ $\mathrm{MHz}, \mathrm{CDCl}_{3}$ ): $\delta=1.05$ (t, $J=7.2 \mathrm{~Hz}, 3 \mathrm{H}$ ), 1.98 (s, $3 \mathrm{H}$ ), 2.30 (s, $3 \mathrm{H}$ ), 4.08 (q, $J=7.2 \mathrm{~Hz}, 2$ $\mathrm{H}), 5.57$ (s, $1 \mathrm{H}), 6.64-6.67$ (m, $2 \mathrm{H}), 6.84-6.86$ (m, $2 \mathrm{H}), 7.09-7.13$ (m, $5 \mathrm{H}) ;{ }^{13} \mathrm{C}$ NMR (90.6 MHz, $\left.\mathrm{CDCl}_{3}\right): \delta=10.7,11.2,14.1,59.4,112.6,115.6,116.6,127.3,127.6,129.9$, 131.0, 131.2, 131.3, 133.0, 138.4, 155.3, 166.3; IR (cm $\left.{ }^{-1}\right)$ : 3357 (m), 2924 (m), 1673 (s), 1517 (s), 1446 (m), 1264 (m); LRMS (EI): 335 (24\%) [M+'], 260 (38\%), 215 (16\%), 141 (32\%), 105 (100\%), 99 (31\%), 71 (34\%), 57 (51\%), 43 (47\%); HRMS 335.1520 [335.1521 calcd for $\left.\mathrm{C}_{21} \mathrm{H}_{21} \mathrm{NO}_{3}\left(\mathrm{M}^{+}\right)\right]$.

\section{Ethyl 4,5-dimethyl-2-phenyl-1-(4-bromophenyl)-pyrrole-3-carboxylate (3ae)}

Following the general procedure, 3af was obtained as a colorless solid (74\%) after flash chromatography on neutral $\mathrm{Al}_{2} \mathrm{O}_{3}(\mathrm{P} /$ EtOAc $=95 / 5) . \mathrm{R}_{\mathrm{f}}=0.75(\mathrm{P} / \mathrm{EtOAc}=80 / 20) ;{ }^{1} \mathrm{H} \mathrm{NMR}$ (360 MHz, $\left.\mathrm{CDCl}_{3}\right): \delta=1.04(\mathrm{t}, J=7.2 \mathrm{~Hz}, 3 \mathrm{H}), 2.00$ (s, $\left.3 \mathrm{H}\right), 2.31(\mathrm{~s}, 3 \mathrm{H}), 4.08$ (q, $J=7.2$ $\mathrm{Hz}, 2 \mathrm{H}), 6.88-6.90$ (m, $2 \mathrm{H}), 7.08-7.18$ (m, $5 \mathrm{H}), 7.38-7.40$ (m, $2 \mathrm{H}) ;{ }^{13} \mathrm{C}$ NMR (90.6 $\left.\mathrm{MHz}, \mathrm{CDCl}_{3}\right): \delta=10.7,11.1,14.1,59.3,113.4,117.2,121.7,127.1,127.5,127.5,130.4$, 131.2, 132.1, 132.6, 137.5, 137.8, 165.8; IR ( $\left.\mathrm{cm}^{-1}\right)$ : 2925 (m), 1694 (s), 1528 (m), 1492 (m),

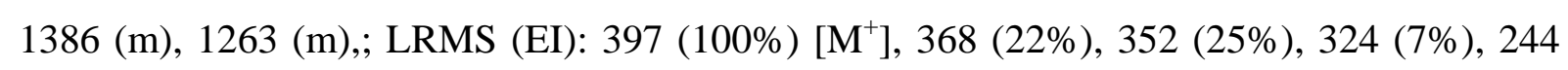
(34\%), 230 (10\%); HRMS 397.0673 [397.0678 calcd for $\mathrm{C}_{21} \mathrm{H}_{20} \mathrm{NO}_{2} \mathrm{Br}\left(\mathrm{M}^{+}\right)$].

\section{Ethyl 4,5-dimethyl-2-phenyl-1-(2-iso-propylphenyl)-pyrrole-3-carboxylate (3ag)}

Following the general procedure, 3ag was obtained as a colorless solid (67\%) after flash chromatography on neutral $\mathrm{Al}_{2} \mathrm{O}_{3}(\mathrm{P} /$ EtOAc $=98 / 2) . \mathrm{R}_{\mathrm{f}}=0.72(\mathrm{P} / \mathrm{EtOAc}=80 / 20) ;{ }^{1} \mathrm{H} \mathrm{NMR}$ (360 MHz, $\left.\mathrm{CDCl}_{3}\right): \delta=0.68$ (d, $\left.J=7.0 \mathrm{~Hz}, 3 \mathrm{H}\right), 0.98$ (d, $\left.J=7.0 \mathrm{~Hz}, 3 \mathrm{H}\right), 1.06$ (t, $J=7.2$ Hz, $3 \mathrm{H}), 1.90$ (s, $3 \mathrm{H}), 2.30-2.39$ (m, $4 \mathrm{H}), 4.04-4.18(\mathrm{~m}, 2 \mathrm{H}), 7.10-7.23(\mathrm{~m}, 8 \mathrm{H})$, 7.27-7.31 (m, $1 \mathrm{H}) ;{ }^{13} \mathrm{C}$ NMR (90.6 $\left.\mathrm{MHz} \mathrm{CDCl}_{3}\right): \delta=10.6,11.3,14.1$, 22.7, 24.6, 27.4, 59.3, 112.6, 116.7, 125.9, 126.7, 127.1, 127.3, 127.6, 129.2, 130.1, 131.3, 132.4, 135.7, 138.0, 146.9, 166.2; IR (cm $\left.{ }^{-1}\right): 2963$ (m), 1698 (s), 1493 (m), 1383 (m), 1235 (m); LRMS (EI): 361 (100\%) [M $\mathrm{M}^{+}$, 346 (24\%), 288 (44\%), 258 (20\%); HRMS 361.2040 [361.2042 calcd for $\left.\mathrm{C}_{24} \mathrm{H}_{27} \mathrm{NO}_{2}\left(\mathrm{M}^{+}\right)\right]$. 


\section{Ethyl 4,5-dimethyl-2-phenyl-1-(3-nitrophenyl)-pyrrole-3-carboxylate (3ah)}

Following the general procedure, 3ah was obtained as a yellow solid (55\%) after flash chromatography on neutral $\mathrm{Al}_{2} \mathrm{O}_{3}(\mathrm{P} /$ EtOAc $=96 / 4) . \mathrm{R}_{\mathrm{f}}=0.48(\mathrm{P} / \mathrm{EtOAc}=80 / 20) ;{ }^{1} \mathrm{H} \mathrm{NMR}$ (360 MHz, $\mathrm{CDCl}_{3}$ ): $\delta=1.04$ (t, $J=7.2 \mathrm{~Hz}, 3 \mathrm{H}$ ), 2.04 (s, $3 \mathrm{H}$ ), 2.33 (s, $3 \mathrm{H}$ ), 4.09 (q, $J=7.2$ Hz, 2 H), 7.08-7.11 (m, 2 H), 7.16-7.18 (m, 3 H), 7.41-7.46 (m, 2 H), 7.93 (s, 1 H), 8.09-8.12 (m, $1 \mathrm{H}) ;{ }^{13} \mathrm{C}$ NMR (90.6 MHz, $\left.\mathrm{CDCl}_{3}\right): \delta=10.8,11.1,14.1$, 59.5, 114.1, 117.9, 122.7, 123.9, 127.0, 127.7, 127.8, 129.7, 131.2, 132.2, 134.8, 137.9, 139.6, 148.4, 165.6; IR

(cm ${ }^{-1}$ ): 2925 (s), 1698 (vs), 1538 (vs), 1483 (m), 1349 (vs), 1238 (m); LRMS (EI): 364 (100\%) $\left[\mathrm{M}^{+}\right], 335$ (21\%), 319 (22\%), 273 (12\%); HRMS 364.1413 [364.1423 calcd for $\left.\mathrm{C}_{21} \mathrm{H}_{20} \mathrm{~N}_{2} \mathrm{O}_{2}\left(\mathrm{M}^{+}\right)\right]$.

\section{Ethyl 4,5-dimethyl-2-phenyl-1-(methyl thiophenyl-2-carboxylate-3-yl)-pyrrole-3- carboxylate (3ai)}

Following the general procedure, 3ai was obtained as a colorless solid (52\%) after flash chromatography on neutral $\mathrm{Al}_{2} \mathrm{O}_{3}(\mathrm{P} /$ EtOAc $=95 / 5) . \mathrm{R}_{\mathrm{f}}=0.42(\mathrm{P} /$ EtOAc $=80 / 20) ;{ }^{1} \mathrm{H} \mathrm{NMR}$ (360 MHz, $\left.\mathrm{CDCl}_{3}\right): \delta=1.04$ (t, $J=7.0 \mathrm{~Hz}, 3 \mathrm{H}$ ), 1.96 (s, $\left.3 \mathrm{H}\right), 2.32$ (s, $\left.3 \mathrm{H}\right), 3.75$ (s, $3 \mathrm{H}$ ), 4.10 (q, $J=7.0 \mathrm{~Hz}, 2 \mathrm{H}), 6.76-6.80(\mathrm{~m}, 1 \mathrm{H}), 7.08-7.17$ (m, $5 \mathrm{H}), 7.36-7.37(\mathrm{~m}, 1 \mathrm{H}) ;{ }^{13} \mathrm{C}$ NMR (90.6 MHz, $\left.\mathrm{CDCl}_{3}\right): \delta=10.2,11.3,14.1,52.4,59.2,113.3,117.1,127.1,127.2,127.5$, 128.1, 128.1, 128.6, 129.7, 130.7, 132.8, 138.1, 140.8, 160.8, 165.8; IR (cm $\left.{ }^{-1}\right): 2924$ (m), 1698 (s), 1437 (m), 1259 (s), 1071 (m); LRMS (EI): 383 (65\%) [M+1, 310 (25\%), 258 (26\%), 213 (14\%), 105 (100\%); HRMS 383.1193 [383.1191 calcd for $\mathrm{C}_{21} \mathrm{H}_{21} \mathrm{NO}_{4} \mathrm{~S}\left(\mathrm{M}^{+}\right)$].

\section{Ethyl 4,5-dimethyl-2-phenyl-1-naphtylpyrrole-3-carboxylate (3aj)}

Following the general procedure, 3aj was obtained as a yellow solid (72\%) after flash chromatography on neutral $\mathrm{Al}_{2} \mathrm{O}_{3}(\mathrm{P} / \mathrm{EtOAc}=90 / 10) . \mathrm{R}_{\mathrm{f}}=0.67(\mathrm{P} / \mathrm{EtOAc}=80 / 20) ;{ }^{1} \mathrm{H} \mathrm{NMR}$ (360 MHz, $\left.\mathrm{CDCl}_{3}\right): \delta=1.05$ (t, $\left.J=7.2 \mathrm{~Hz}, 3 \mathrm{H}\right), 1.82$ (s, $\left.3 \mathrm{H}\right), 2.39$ (s, $\left.3 \mathrm{H}\right), 4.11$ (q, $J=7.2$ Hz, 2 H), 6.95-7.01 (m, 3 H), 7.06-7.09 (m, 2 H), 7.20-7.22 (m, 1 H), 7.31-7.37 (m, 2 H), 7.44-7.50 (m, $2 \mathrm{H}), 7.76-7.83$ (m, $2 \mathrm{H}$ ); ${ }^{13} \mathrm{C}$ NMR (90.6 MHz, $\mathrm{CDCl}_{3}$ ): $\delta=10.2$, 11.3, 14.1, 59.3, 113.0, 116.7, 123.3, 125.1, 126.6, 127.0, 127.2, 127.3, 127.4, 128.2, 128.4, 128.9, 130.7, 131.8, 132.9, 133.9, 135.1, 139.2, 166.1; IR (cm $\left.{ }^{-1}\right): 2977$ (w), 1696 (s), 1598 (w), 1508 (w), 1379 (w), 1254 (m), 1150 (m), 1069 (m); LRMS (EI): 369 (100\%) [M+1, 340 (20\%), 324 (18\%), 167 (12\%), 149 (16\%), 127 (23\%), 105 (24\%); HRMS 369.1720 [369.1729 calcd for $\left.\mathrm{C}_{25} \mathrm{H}_{23} \mathrm{NO}_{2}\left(\mathrm{M}^{+}\right)\right]$. 
Ethyl 4,5-dimethyl-2-phenyl-1-(4-[Ethyl 4,5-dimethyl-2-phenyl-pyrrole-3-carboxylate-1yl]-phenyl)-pyrrole-3-carboxylate (3ak)

Following the general procedure, 3ak was obtained as a yellow solid (31\%) after flash chromatography on neutral $\mathrm{Al}_{2} \mathrm{O}_{3}(\mathrm{P} /$ EtOAc $=95 / 5) . \mathrm{R}_{\mathrm{f}}=0.33(\mathrm{P} /$ EtOAc $=80 / 20) ;{ }^{1} \mathrm{H} \mathrm{NMR}$ $\left(360 \mathrm{MHz}, \mathrm{CDCl}_{3}\right): \delta=1.04(\mathrm{t}, J=7.0 \mathrm{~Hz}, 6 \mathrm{H}), 1.93$ (s, $\left.6 \mathrm{H}\right), 2.29$ (s, $\left.6 \mathrm{H}\right), 4.08$ (q, $J=7.0$ $\mathrm{Hz}, 4 \mathrm{H}), 6.89$ (s, $4 \mathrm{H}), 7.00-7.02$ (m, $4 \mathrm{H}), 7.09-7.14$ (m, $6 \mathrm{H}) ;{ }^{13} \mathrm{C}$ NMR (90.6 MHz, $\left.\mathrm{CDCl}_{3}\right): \delta=10.6,11.1,14.1,59.4,113.3,117.2,127.1,127.4,127.4,129.1,131.1,132.5$, 137.7, 137.8, 165.8; IR (cm $\left.{ }^{-1}\right): 2924$ (s), 2851 (w), 1694 (s), 1519 (m), 1480 (m), 1381 (m), 1255 (m), 1154 (m), 1069 (m); LRMS (EI): 560 (16\%) [M+'], 233 (6\%), 105 (100\%); HRMS $560.2683\left[560.2675\right.$ calcd for $\left.\mathrm{C}_{36} \mathrm{H}_{36} \mathrm{~N}_{2} \mathrm{O}_{4}\left(\mathrm{M}^{+}\right)\right]$.

\section{Ethyl 2,5-dimethyl-4-phenyl-1-phenyl-pyrrole-3-carboxylate (3ba) ${ }^{4}$}

Following the general procedure, 3ba was obtained as a yellow oil (75\%) after flash chromatography on neutral $\mathrm{Al}_{2} \mathrm{O}_{3}(\mathrm{P} /$ EtOAc $=96 / 4) . \mathrm{R}_{\mathrm{f}}=0.73(\mathrm{P} / \mathrm{EtOAc}=80 / 20) ;{ }^{1} \mathrm{H} \mathrm{NMR}$ (500 MHz, $\left.\mathrm{CDCl}_{3}\right): \delta=1.03(\mathrm{t}, J=7.2 \mathrm{~Hz}, 3 \mathrm{H}), 1.89$ (s, $\left.3 \mathrm{H}\right), 2.32$ (s, $\left.3 \mathrm{H}\right), 4.09$ (q, $J=7.2$ $\mathrm{Hz}, 2 \mathrm{H}), 7.24-7.24$ (m,1 H), 7.27-7.39 (m, $6 \mathrm{H}), 7.43-7.56$ (m, $3 \mathrm{H}) ;{ }^{13} \mathrm{C}$ NMR (90.6 MHz, $\left.\mathrm{CDCl}_{3}\right): \delta=11.4,12.7,14.1,59.3,111.2,122.4,126.0,126.9,127.5,128.4,128.7,129.6$, 130.6, 135.9, 136.6, 138.0, 166.1; IR (cm $\left.{ }^{-1}\right): 2923$ (m), 1694 (s), 1598 (w), 1497 (m).

\section{Ethyl 2,5-dimethyl-4-phenyl-1-[4-methoxyphenyl]-pyrrole-3-carboxylate (3bb)}

Following the general procedure, 3bb was obtained as a yellow oil (75\%) from after flash chromatography on neutral $\mathrm{Al}_{2} \mathrm{O}_{3}(\mathrm{P} /$ EtOAc $=95 / 5) . \mathrm{R}_{\mathrm{f}}=0.53(\mathrm{P} / \mathrm{EtOAc}=80 / 20) ;{ }^{1} \mathrm{H} \mathrm{NMR}$ (500 MHz, $\left.\mathrm{CDCl}_{3}\right): \delta=1.03$ (t, $\left.J=7.1 \mathrm{~Hz}, 3 \mathrm{H}\right), 1.88$ (s, $\left.3 \mathrm{H}\right), 2.31$ (s, $\left.3 \mathrm{H}\right), 3.88$ (s, $\left.3 \mathrm{H}\right)$, 4.08 (q, $J=7.1 \mathrm{~Hz}, 2 \mathrm{H}), 7.00-7.02$ (m, $2 \mathrm{H}), 7.16-7.17$ (m, $2 \mathrm{H}), 7.23-7.25$ (m, $1 \mathrm{H})$, 7.29-7.30 (m, $2 \mathrm{H}), 7.33-7.36$ (m, $2 \mathrm{H}) ;{ }^{13} \mathrm{C} \mathrm{NMR}\left(90.6 \mathrm{MHz}, \mathrm{CDCl}_{3}\right): \delta=11.3,12.7,14.1$, 55.7, 59.2, 111.0, 114.7, 122.2, 126.0, 127.2, 127.5, 129.4, 130.6, 130.7, 136.2, 136.7, 159.7, 166.1; IR (cm ${ }^{-1}$ ): 2922 (m), 1690 (s), 1606 (m), 1514 (s), 1442 (w), 1250 (m), 1179 (m), 1082

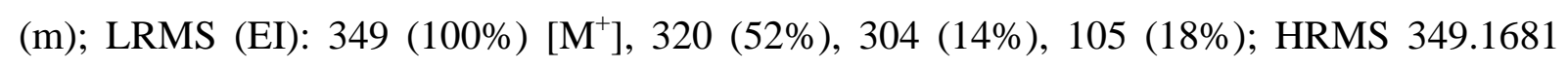
[349.1678 calcd for $\mathrm{C}_{22} \mathrm{H}_{23} \mathrm{NO}_{3}\left(\mathrm{M}^{+}\right)$].

\section{Ethyl 2,5-dimethyl-4-[2-methoxyphenyl]-1-phenylpyrrole-3-carboxylate (3ca)}

Following the general procedure, 3ca was obtained as a yellow solid (83\%) after flash chromatography on neutral $\mathrm{Al}_{2} \mathrm{O}_{3}(\mathrm{P} /$ EtOAc $=95 / 5) . \mathrm{R}_{\mathrm{f}}=0.45(\mathrm{P} / \mathrm{EtOAc}=80 / 20) ;{ }^{1} \mathrm{H} \mathrm{NMR}$

\footnotetext{
${ }^{4}$ Huisgen, R.; Gotthardt, H.; Bayer, H. O. Angew. Chem. 1964, 76, 185.
} 
(360 MHz, $\left.\mathrm{CDCl}_{3}\right): \delta=0.98$ (t, $\left.J=7.0 \mathrm{~Hz}, 3 \mathrm{H}\right), 1.87$ (s, $\left.3 \mathrm{H}\right), 2.31$ (s, $\left.3 \mathrm{H}\right), 3.79$ (s, $3 \mathrm{H}$ ), 4.05 (q, $J=7.0 \mathrm{~Hz}, 2 \mathrm{H}), 6.91-6.99$ (m, $2 \mathrm{H}), 7.19-7.24$ (m, $2 \mathrm{H}), 7.29-7.33$ (m, $2 \mathrm{H})$,

7.42-7.52 (m, $3 \mathrm{H}) ;{ }^{13} \mathrm{C}$ NMR (90.6 $\left.\mathrm{MHz} \mathrm{CDCl}_{3}\right): \delta=11.5,12.7,14.1,55.6,59.1,110.4$, 112.1, 117.9, 120.1, 125.7, 127.1, 127.7, 128.5, 128.5, 129.4, 131.9, 135.3, 138.2, 157.7, 166.3; IR (cm $\left.{ }^{-1}\right)$ : 2925 (m), 1694 (s), 1597 (w), 1538 (m), 1494 (m); LRMS (EI): 349 (100\%) $\left[\mathrm{M}^{+}\right.$], 320 (34\%), 287 (19\%), 276 (53\%); HRMS 349.1680 [349.1678 calcd for $\mathrm{C}_{22} \mathrm{H}_{23} \mathrm{NO}_{3}$ $\left.\left(\mathrm{M}^{+}\right)\right]$.

\section{Methyl 4,5-dimethyl-2-(n-pentyl)-1-phenylpyrrole-3-carboxylate (3da)}

Following the general procedure, 3da was obtained as a colorless liquid (68\%) after flash chromatography on neutral $\mathrm{Al}_{2} \mathrm{O}_{3}(\mathrm{P} /$ EtOAc $=98 / 2) . \mathrm{R}_{\mathrm{f}}=0.77(\mathrm{P} /$ EtOAc $=80 / 20) ;{ }^{1} \mathrm{H} \mathrm{NMR}$ (360 MHz, $\left.\mathrm{CDCl}_{3}\right): \delta=0.76(\mathrm{t}, J=6.9 \mathrm{~Hz}, 3 \mathrm{H}), 1.05-1.18(\mathrm{~m}, 4 \mathrm{H}), 1.33-1.41(\mathrm{~m}, 2 \mathrm{H})$, 1.85 (s, 3 H), 2.23 (s, 3 H), 2.62-2.66 (m, 2 H), 3.81 (s, 3 H), 7.16-7.19 (m, 2 H), 7.44-7.50 (m, $3 \mathrm{H}) ;{ }^{13} \mathrm{C}$ NMR (90.6 MHz, $\left.\mathrm{CDCl}_{3}\right): \delta=10.4,11.4,14.0,22.2,26.2,30.0,31.8,50.5$, 110.4, 115.9, 125.8, 128.6, 128.8, 129.3, 128.3, 141.0, 166.8; IR (cm-1): 2928 (s), 2851 (m), 1718 (s), 1503 (m), 1438 (m), 1252 (m), 1140 (m); LRMS (EI): 299 (30\%) [M+1, 268 (4\%), 242 (100\%), 182 (8\%); HRMS 299.1885 [299.1885 calcd for $\mathrm{C}_{19} \mathrm{H}_{25} \mathrm{NO}_{2}\left(\mathrm{M}^{+}\right)$].

\section{Ethyl 2,4-diphenyl-1-phenylpyrrole-3-carboxylate (3ea)}

Following the general procedure, 3ea was obtained as a yellow solid (65\% [72\% starting from $1.3 \mathrm{mmol}$ of $\mathbf{1 k}$ ]) after flash chromatography on neutral $\mathrm{Al}_{2} \mathrm{O}_{3}(\mathrm{P} /$ EtOAc $=95 / 5) . \mathrm{R}_{\mathrm{f}}=0.63$ $(\mathrm{P} / \mathrm{EtOAc}=80 / 20) ;{ }^{1} \mathrm{H}$ NMR $\left(360 \mathrm{MHz}, \mathrm{CDCl}_{3}\right): \delta=0.86(\mathrm{t}, J=7.0 \mathrm{~Hz}, 3 \mathrm{H}), 2.04(\mathrm{~s}, 3 \mathrm{H})$, 3.96 (q, $J=7.0 \mathrm{~Hz}, 2 \mathrm{H}), 7.12-7.15$ (m, 2 H), 7.18-7.20 (m, $3 \mathrm{H}), 7.23-7.26$ (m, $2 \mathrm{H})$, 7.29-7.35 (m, $4 \mathrm{H}), 7.39-7.43$ (m, $4 \mathrm{H}$ ); ${ }^{13} \mathrm{C} \mathrm{NMR}\left(90.6 \mathrm{MHz}, \mathrm{CDCl}_{3}\right): \delta=11.7,13.7,59.6$, 113.3, 123.2, 126.3, 127.4, 127.5, 127.8, 128.0, 128.2, 128.8, 129.0, 130.5, 131.2, 132.2, 136.1, 137.7, 138.2, 165.5; IR (cm $\left.{ }^{-1}\right): 2921$ (m), 1698 (s), 1597 (w), 1495 (m), 1440 (m), 1381

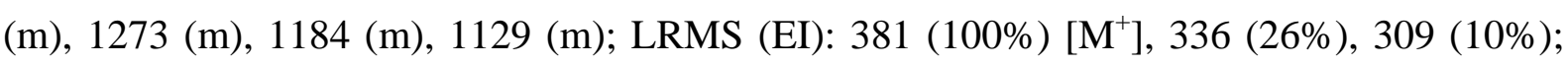
HRMS 381.1732 [381.1729 calcd for $\mathrm{C}_{26} \mathrm{H}_{23} \mathrm{NO}_{2}\left(\mathrm{M}^{+}\right)$].

\section{Ethyl 5-methyl-2-phenyl-4-(thiophen-3-yl)-1-phenylpyrrole-3-carboxylate (3fa)}

Following the general procedure, 3fa was obtained as a colorless solid (74\%) after flash chromatography on neutral $\mathrm{Al}_{2} \mathrm{O}_{3}(\mathrm{P} /$ EtOAc $=95 / 5) . \mathrm{R}_{\mathrm{f}}=0.69(\mathrm{P} /$ EtOAc $=80 / 20) ;{ }^{1} \mathrm{H} \mathrm{NMR}$ (360 MHz, $\left.\mathrm{CDCl}_{3}\right): \delta=0.90$ (t, $J=7.2 \mathrm{~Hz}, 3 \mathrm{H}$ ), 2.05 (s, $3 \mathrm{H}$ ), 3.98 (q, $J=7.2 \mathrm{~Hz}, 2 \mathrm{H}$ ), 7.11 (d, $2 \mathrm{H}), 7.16-7.21$ (m, $6 \mathrm{H}), 7.22-7.24$ (m, $1 \mathrm{H}), 7.27-7.34$ (m, $4 \mathrm{H}) ;{ }^{13} \mathrm{C}$ NMR (90.6 MHz, 
$\mathrm{CDCl}_{3}$; one $\mathrm{C}_{\mathrm{Ar}}$ missing): $\delta=11.9,13.8,59.6,113.4,117.7,122.6,123.8,127.4,127.5,128.1$, 128.8, 129.0, 130.5, 131.1, 132.2, 135.7, 137.7, 138.1, 165.5; IR (cm $\left.{ }^{-1}\right)$ : 2923 (m), 1703 (vs), 1598 (w), 1498 (m), 1400 (m), 1263 (m), 1210 (m), 1132 (s); LRMS (EI): 387 (100\%) [M+1, 342 (26\%), 312 (12\%), 277 (9\%); HRMS 387.1292 [387.1293 calcd for $\mathrm{C}_{24} \mathrm{H}_{21} \mathrm{NO}_{2} \mathrm{~S}\left(\mathrm{M}^{+}\right)$].

\section{Ethyl 4-cyclohexylmethyl-5-methyl-2-phenyl-1-phenylpyrrole-3-carboxylate (3ga)}

Following the general procedure, 3ga was obtained as a colorless solid (90\%) after flash chromatography on neutral $\mathrm{Al}_{2} \mathrm{O}_{3}(\mathrm{P} /$ EtOAc $=95 / 5) . \mathrm{R}_{\mathrm{f}}=0.84(\mathrm{P} / \mathrm{EtOAc}=80 / 20) ;{ }^{1} \mathrm{H} \mathrm{NMR}$ (360 MHz, $\left.\mathrm{CDCl}_{3}\right): \delta=0.97-1.00$ (m, $\left.2 \mathrm{H}\right), 1.06$ (t, $\left.J=7.2 \mathrm{~Hz}, 3 \mathrm{H}\right), 1.15-1.22(\mathrm{~m}, 3 \mathrm{H})$, 1.60-1.73 (m, 4 H), 1.77-1.81 (m, 2 H), 1.98 (s, 3 H), 2.63 (d, $J=6.8$ Hz, 2 H), 4.09 (q, $J=$ 7.2 Hz, 2 H), 7.00-7.03 (m, 2 H), 7.11-7.17 (m, 5 H), 7.24-7.27 (m, $3 \mathrm{H})$ ) ${ }^{13} \mathrm{C}$ NMR (90.6 $\left.\mathrm{MHz}, \mathrm{CDCl}_{3}\right): \delta=11.3,14.1,26.7,26.9,33.3,33.6,40.0,59.3,112.8,120.5,127.2,127.3$, 127.8, 127.9, 128.8, 128.9, 131.2, 133.1, 138.1, 138.5, 166.0; IR ( $\left.\mathrm{cm}^{-1}\right)$ : 2922 (s), $2848(\mathrm{w})$, 1698 (s), 1498 (m), 1384 (m), 1234 (m), 1111 (s); LRMS (EI): 401 (25\%) [M'], 326 (99\%), 267 (28\%), 244 (100\%), 215 (26\%); HRMS 401.2350 [401.2355 calcd for $\mathrm{C}_{27} \mathrm{H}_{31} \mathrm{NO}_{2}\left(\mathrm{M}^{+}\right)$].

\section{Ethyl 4-cyclohexylmethyl-5-methyl-2-phenyl-1-naphtylpyrrole-3-carboxylate (3gj)}

Following the general procedure, 3gj was obtained as a yellow solid (79\%) after flash chromatography on neutral $\mathrm{Al}_{2} \mathrm{O}_{3}(\mathrm{P} /$ EtOAc $=95 / 5) . \mathrm{R}_{\mathrm{f}}=0.72(\mathrm{P} /$ EtOAc $=80 / 20) ;{ }^{1} \mathrm{H} \mathrm{NMR}$ (360 MHz, $\left.\mathrm{CDCl}_{3}\right): \delta=0.99-1.10(\mathrm{~m}, 5 \mathrm{H}), 1.18-1.24(\mathrm{~m}, 2 \mathrm{H}), 1.30-1.37(\mathrm{~m}, 1 \mathrm{H})$, 1.68-1.77 (m, 4 H), 1.82-1.87 (m, 5 H), 2.66 (dd, $J=13.4$ Hz, $J=7.3$ Hz, 1 H), 2.76 (dd, $J$ $=13.4 \mathrm{~Hz}, J=6.6 \mathrm{~Hz}, 1 \mathrm{H}), 4.12(\mathrm{q}, J=7.2 \mathrm{~Hz}, 2 \mathrm{H}), 6.96-7.01$ (m, $3 \mathrm{H}), 7.06-7.08$ (m, 2 H), 7.22-7.34 (m, $1 \mathrm{H}), 7.28-7.37$ (m, $2 \mathrm{H}), 7.46-7.49$ (m, $2 \mathrm{H}), 7.75-7.83$ (m, $2 \mathrm{H}) ;{ }^{13} \mathrm{C}$ NMR (90.6 MHz, $\left.\mathrm{CDCl}_{3}\right): \delta=10.7,14.1,26.7,27.0$, 33.5, 33.8, 39.9, 59.3, 112.7, 120.3, 123.2, 125.0, 126.6, 127.0, 127.2, 127.2, 127.4, 128.2, 128.8, 129.1, 130.6, 131.9, 133.0, 133.9, 135.2, 139.4, 166.0; IR (cm-1): 2922 (s), 2850 (m), 1698 (s), 1598 (w), 1479 (m), 1258

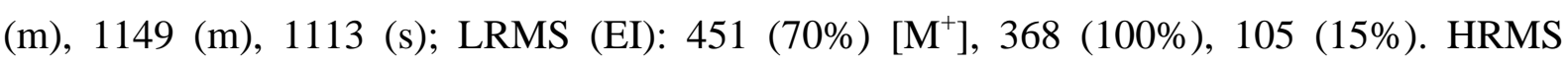
451.2520 [451.2511 calcd for $\left.\mathrm{C}_{31} \mathrm{H}_{33} \mathrm{NO}_{2}\left(\mathrm{M}^{+}\right)\right]$.

\section{Ethyl 4-(3-[\{tetrahydropyran-2-yl\}-oxy]-propyl)-5-methyl-2-phenyl-1-phenylpyrrole-3- carboxylate (3ha)}

Following the general procedure, 3ha was obtained as a colorless solid (70\%) after flash chromatography on neutral $\mathrm{Al}_{2} \mathrm{O}_{3}(\mathrm{P} /$ EtOAc $=95 / 5) . \mathrm{R}_{\mathrm{f}}=0.47(\mathrm{P} / \mathrm{EtOAc}=80 / 20) ;{ }^{1} \mathrm{H} \mathrm{NMR}$ (360 MHz, $\left.\mathrm{CDCl}_{3}\right): \delta=1.05$ (t, $\left.J=7.2 \mathrm{~Hz}, 3 \mathrm{H}\right), 1.56-1.64(\mathrm{~m}, 3 \mathrm{H}), 1.71-1.77(\mathrm{~m}, 1 \mathrm{H})$, 
1.82-197 (m, 4 H), 2.00 (s, 3 H), 2.83-2.87 (m, 2 H), 3.46-3.55 (m, 2 H), 3.81-3.95 (m, 2 H), 4.08 (q, $J=7.2$ Hz, 2 H), 4.60-4.64 (m, 1 H), 7.00-7.03 (m, 2 H), 7.12-7.15 (m, 5 H), 7.22-7.25 (m, $3 \mathrm{H}) ;{ }^{13} \mathrm{C}$ NMR (90.6 $\mathrm{MHz} \mathrm{CDCl}_{3}$; one $\mathrm{C}_{\mathrm{Ar}}$ missing): $\delta=10.8,14.1$, 19.9, 22.2, 25.7, 31.0, 31.4, 59.3, 62.5, 67.5, 99.0, 112.3, 121.1, 127.2, 127.5, 127.8, 128.8, 128.8, 131.2, 132.9, 138.2, 138.3, 165.7; IR (cm ${ }^{-1}$ ): 2940 (s), 1698 (vs), 1598 (m), 1498 (m), 1384

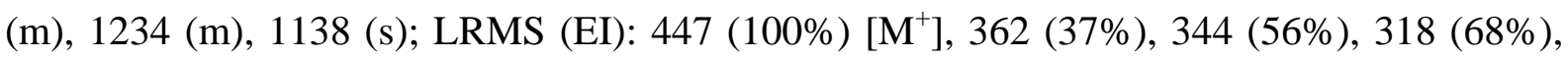
290 (31\%), 274 (23\%); HRMS 447.2401 [447.2410 calcd for $\mathrm{C}_{28} \mathrm{H}_{33} \mathrm{NO}_{4}\left(\mathrm{M}^{+}\right)$].

\section{Ethyl 5-methyl-2-phenyl-1-phenylpyrrole-3-carboxylate (3ia)}

Following the general procedure, 3ia was obtained as a yellow solid (52\%) after flash chromatography on neutral $\mathrm{Al}_{2} \mathrm{O}_{3}(\mathrm{P} /$ EtOAc $=95 / 5) . \mathrm{R}_{\mathrm{f}}=0.60(\mathrm{P} /$ EtOAc $=80 / 20) ;{ }^{1} \mathrm{H} \mathrm{NMR}$ (360 MHz, $\left.\mathrm{CDCl}_{3}\right): \delta=1.16$ (t, $J=7.2 \mathrm{~Hz}, 3 \mathrm{H}$ ), 2.08 (s, $\left.3 \mathrm{H}\right), 4.15$ (q, $\left.J=7.2 \mathrm{~Hz}, 2 \mathrm{H}\right), 6.55$ (s, $1 \mathrm{H}), 7.02-7.04$ (m, $2 \mathrm{H}), 7.15-7.18$ (m, $6 \mathrm{H}), 7.26-7.28$ (m, $2 \mathrm{H}) ;{ }^{13} \mathrm{C}$ NMR $(90.6 \mathrm{MHz}$, $\left.\mathrm{CDCl}_{3}\right): \delta=13.1,14.4$, 59.5, 108.9, 113.2, 127.3, 127.5, 128.0, 128.6, 128.9, 130.3, 131.2, 132.0, 138.1, 138.6, 165.0; IR ( $\left.\mathrm{cm}^{-1}\right)$ : 2923 (m), 1698 (s), 1496 (m), 1422 (m), 1225 (vs), 1112 (s); LRMS (EI): 305 (100\%) [ $\left.\mathrm{M}^{+}\right], 277$ (14\%), 260 (61\%), 217 (12\%), 105 (24\%); HRMS 305.1418 [305.1416 calcd for $\mathrm{C}_{20} \mathrm{H}_{19} \mathrm{NO}_{2}\left(\mathrm{M}^{+}\right)$].

\section{Ethyl 4-(tert-butyldimethylsilyl)-5-methyl-2-phenyl-1-phenylpyrrole-3-carboxylate (3ja)}

Following the general procedure, 3ja was obtained as a colorless solid (77\%) after flash chromatography on neutral $\mathrm{Al}_{2} \mathrm{O}_{3}(\mathrm{P} /$ EtOAc $=98 / 2) . \mathrm{R}_{\mathrm{f}}=0.82(\mathrm{P} / \mathrm{EtOAc}=80 / 20) ;{ }^{1} \mathrm{H} \mathrm{NMR}$ (360 MHz, $\mathrm{CDCl}_{3}$ ): $\delta=0.03$ (s, $6 \mathrm{H}$ ), 0.95 (t, $\left.J=7.2 \mathrm{~Hz}, 3 \mathrm{H}\right), 1.03$ (s, $\left.9 \mathrm{H}\right), 2.13$ (s, $3 \mathrm{H}$ ), 4.01 (q, $J=7.2 \mathrm{~Hz}, 2 \mathrm{H}), 7.04-7.13(\mathrm{~m}, 7 \mathrm{H}), 7.27-7.29(\mathrm{~m}, 3 \mathrm{H}) ;{ }^{13} \mathrm{C}$ NMR $(90.6 \mathrm{MHz}$, $\left.\mathrm{CDCl}_{3}\right): \delta=-2.5,13.9,14.9,18.9,27.5,60.1,111.1,121.3,127.0,127.5,127.9,128.9,129.0$, 130.4, 132.7, 136.9, 137.5, 138.5, 167.9; IR ( $\left.\mathrm{cm}^{-1}\right)$ : 3416 (s), 2951 (s), 1712 (vs), 1499 (s), 1398 (m), 1205 (s), 1132 (s), 1068 (m); LRMS (EI): 419 (5\%) [M+], 362 (100\%), 334 (69\%), 287 (13\%), 131 (17\%); HRMS 419.2281 [418.2281 calcd for $\mathrm{C}_{26} \mathrm{H}_{33} \mathrm{NO}_{2} \mathrm{Si}\left(\mathrm{M}^{+}\right)$]. 

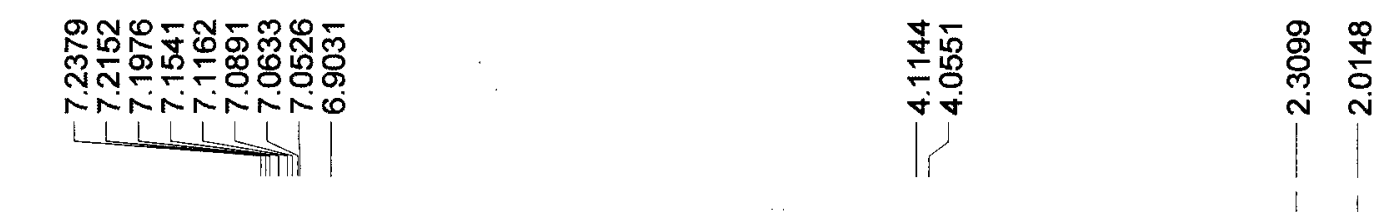

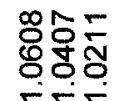

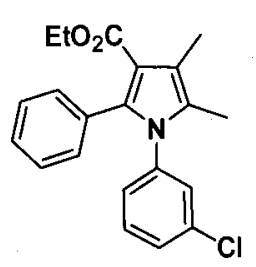

3af

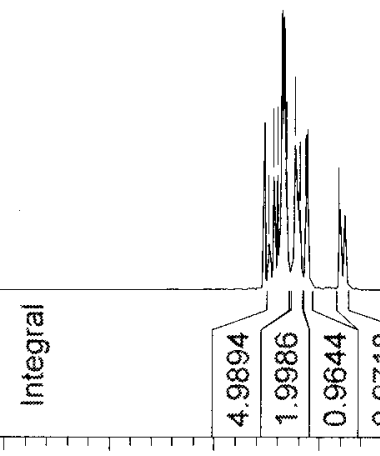

$\begin{array}{lllllll}8.0 & 7.5 & 7.0 & 6.5 & 6.0 & 5.5 & 5.0\end{array}$ (ppm)

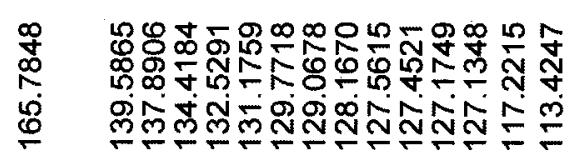

ลิ

穴

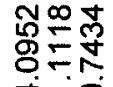

푼
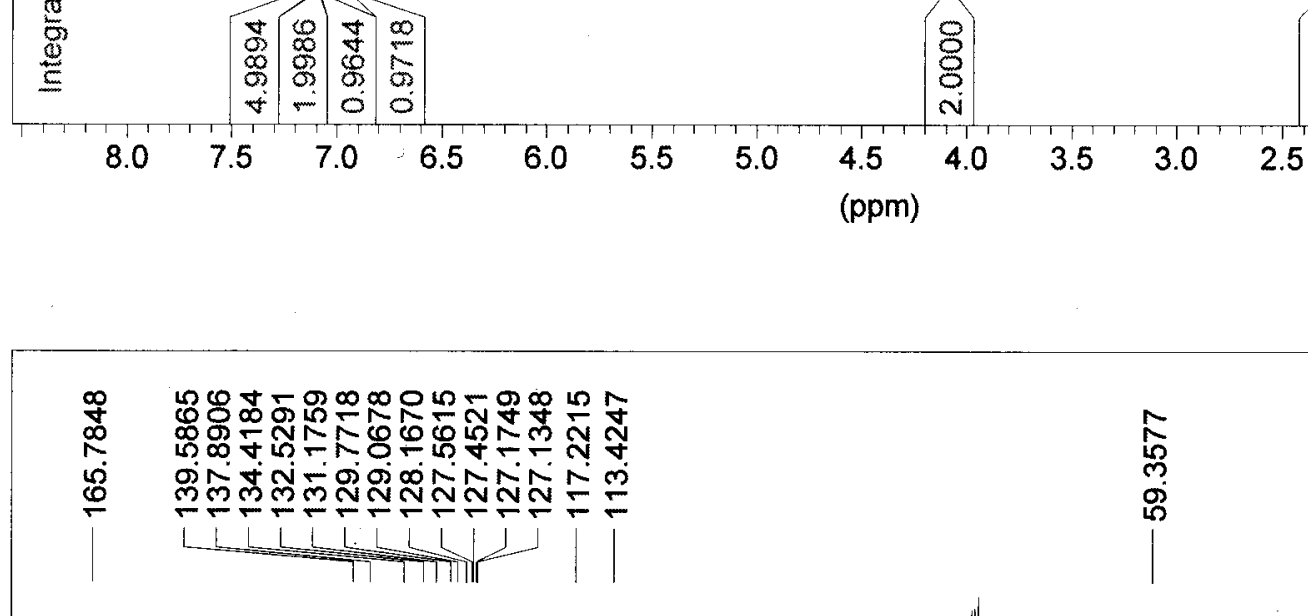

$\left(\begin{array}{c}\mathbb{8} \\ 8 \\ 0 \\ 0\end{array}\right)\left(\begin{array}{l}0 \\ 0 \\ 0 \\ 0 \\ N\end{array}\right)$

$\underset{\square}{\square}$

$1.0 \quad 0.5$

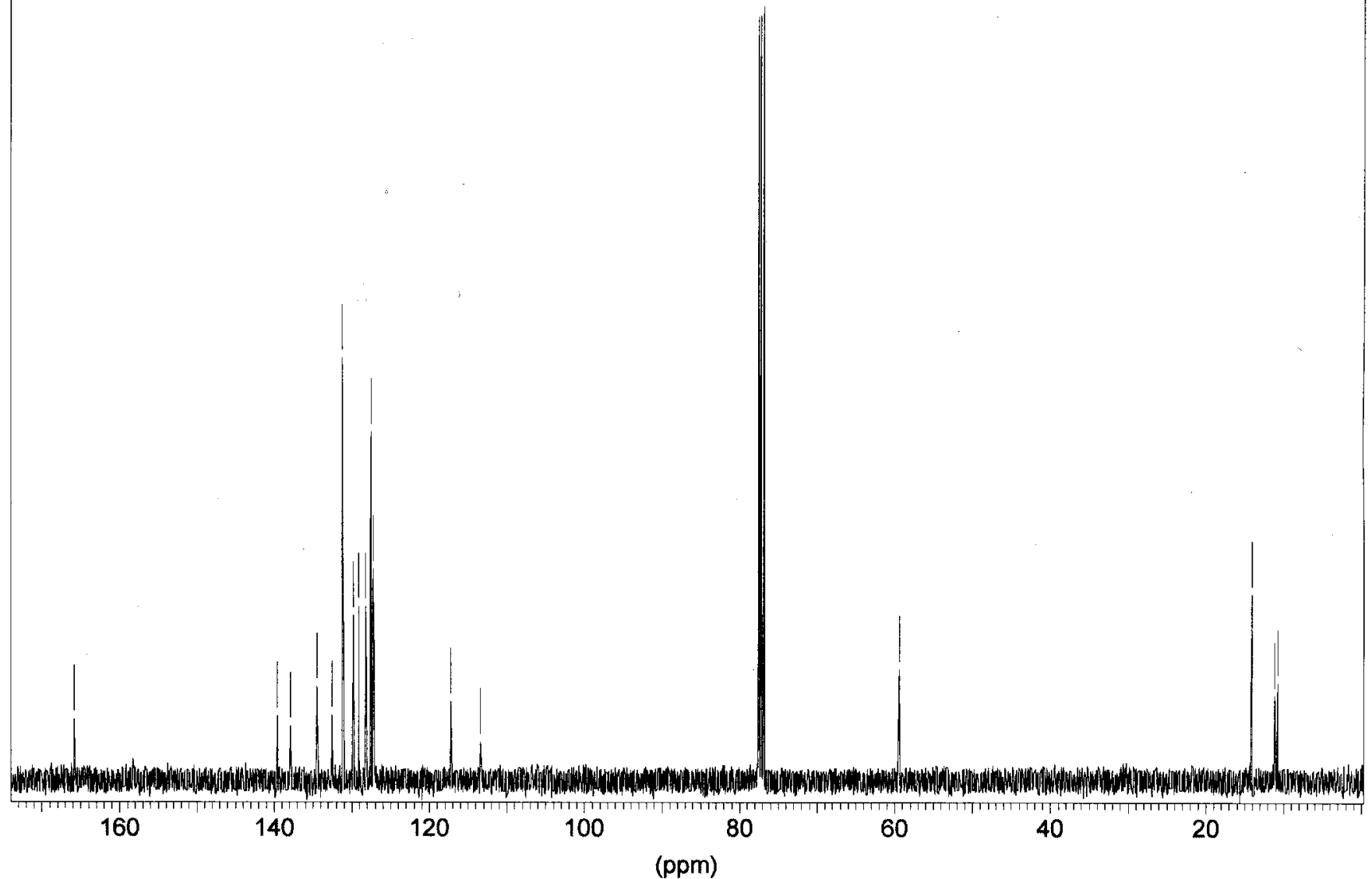


S11

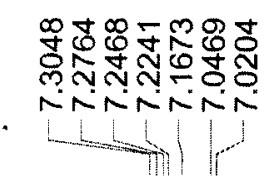

影兽

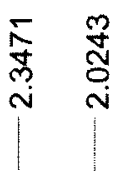

\begin{tabular}{l}
0 \\
0 \\
0 \\
\hdashline
\end{tabular}
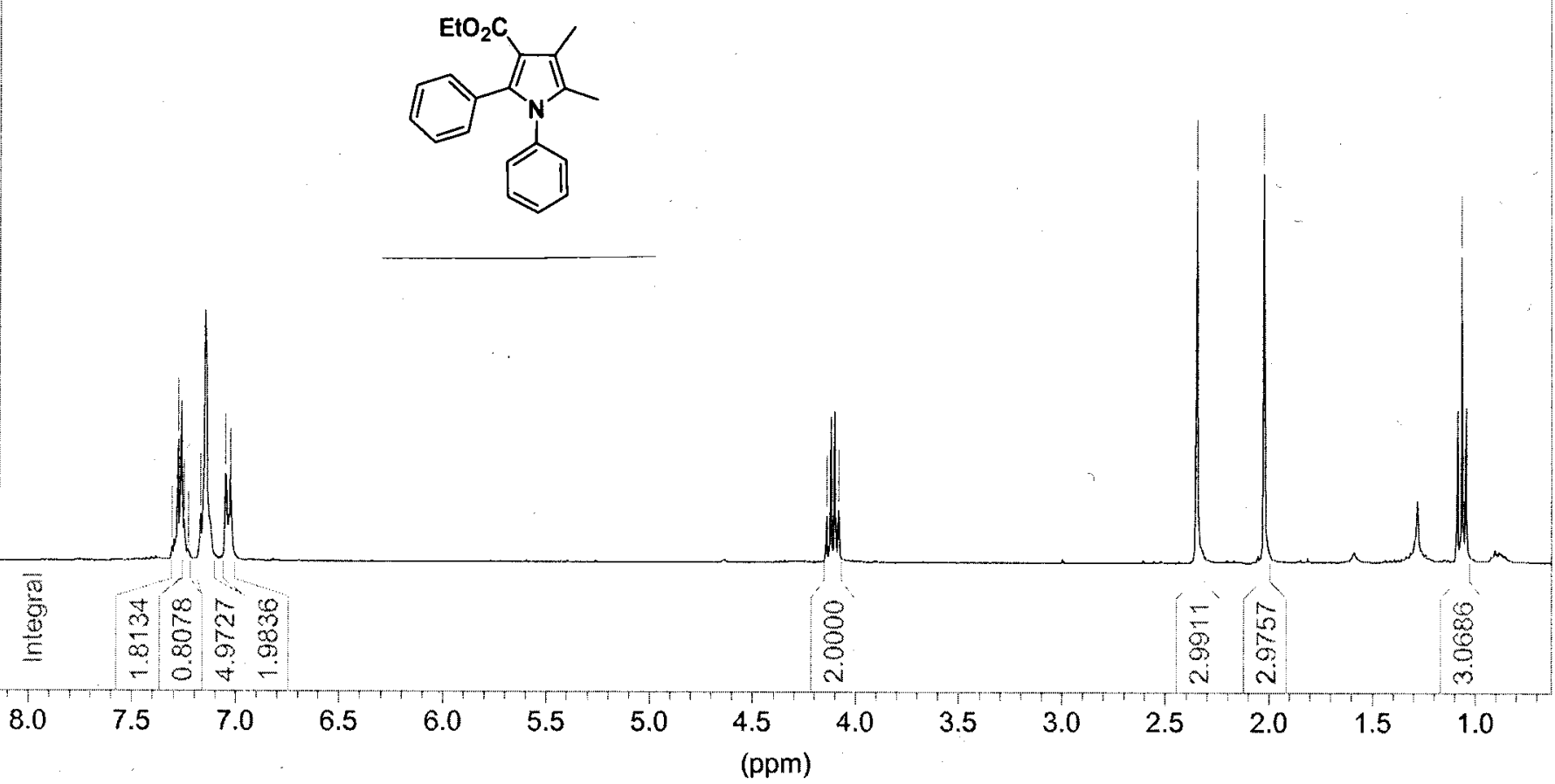

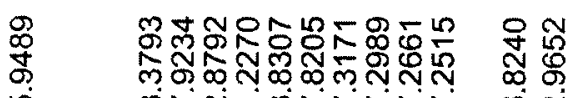

!

$\stackrel{1}{8}$

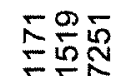

品

$ㅍ ㅜ ㅇ ㅡ$
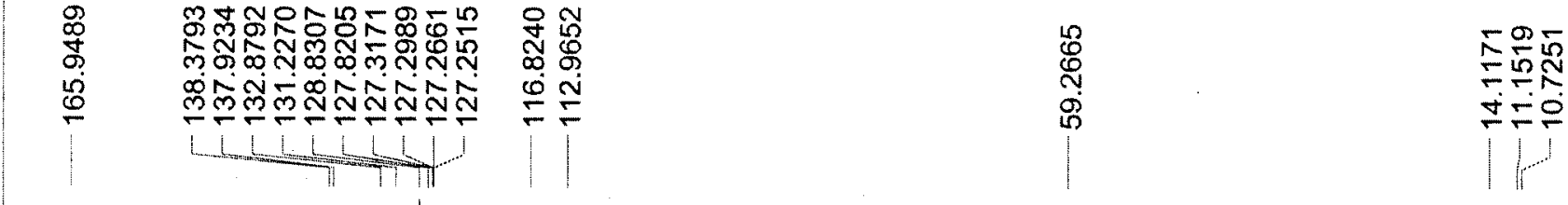

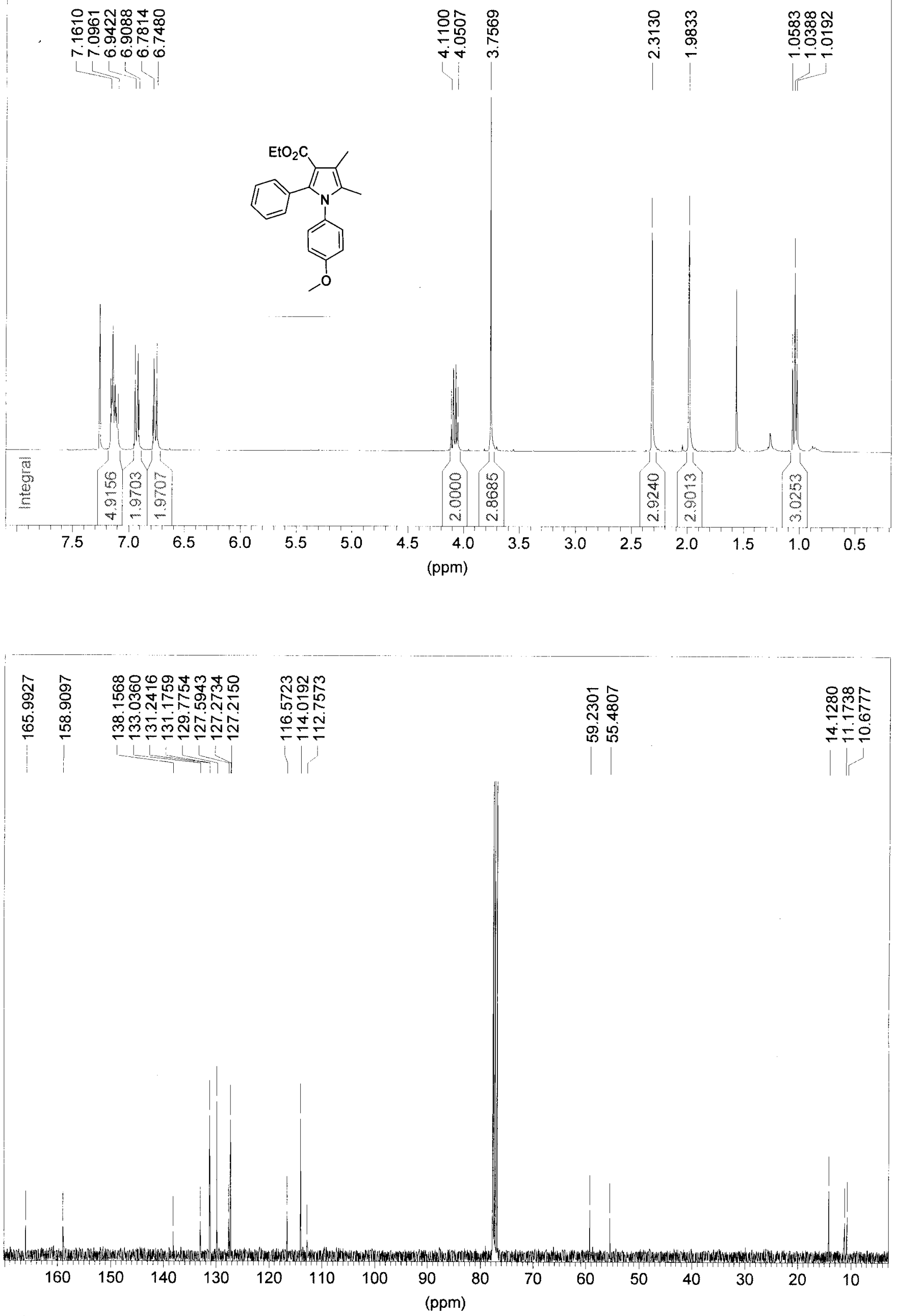

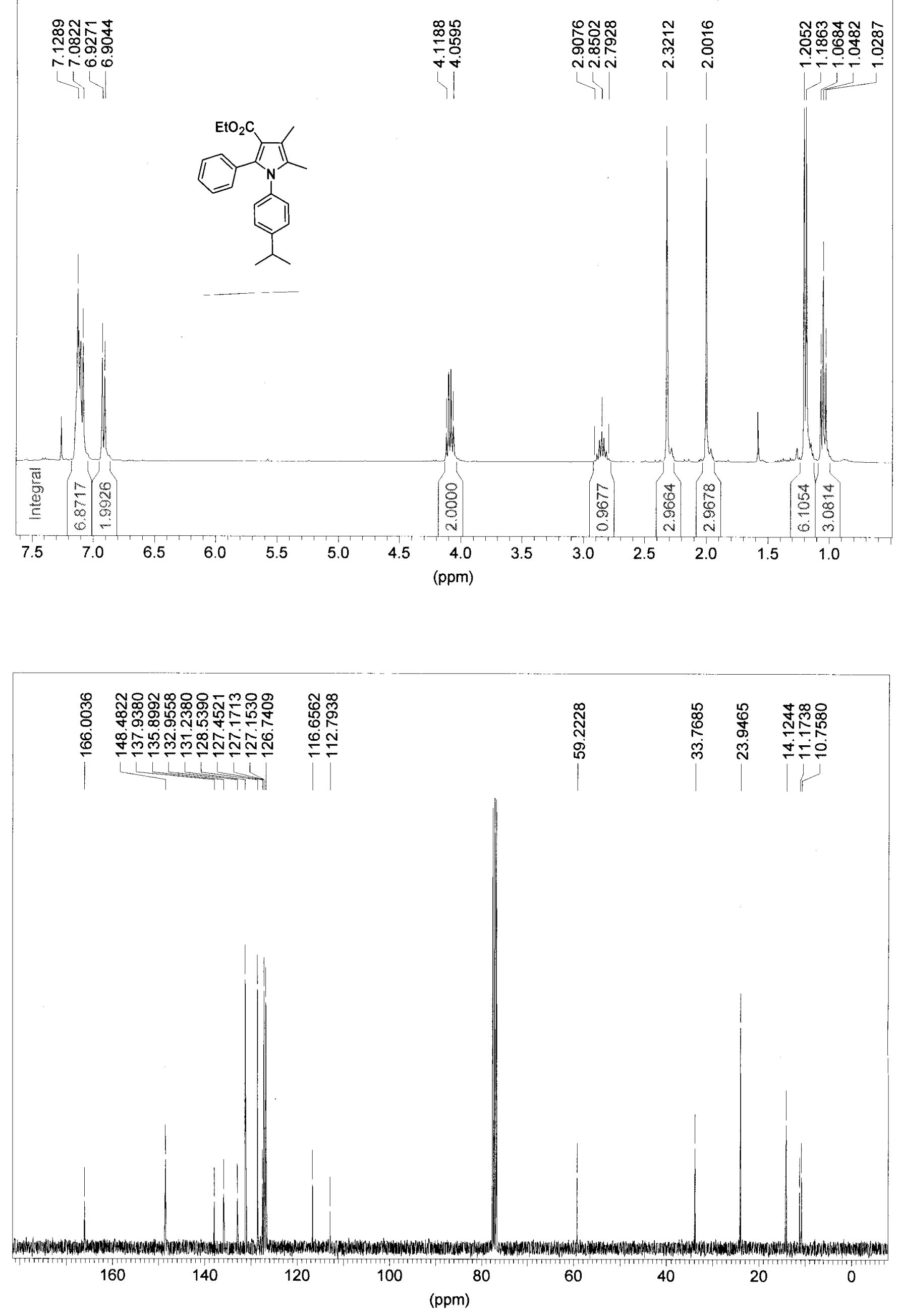

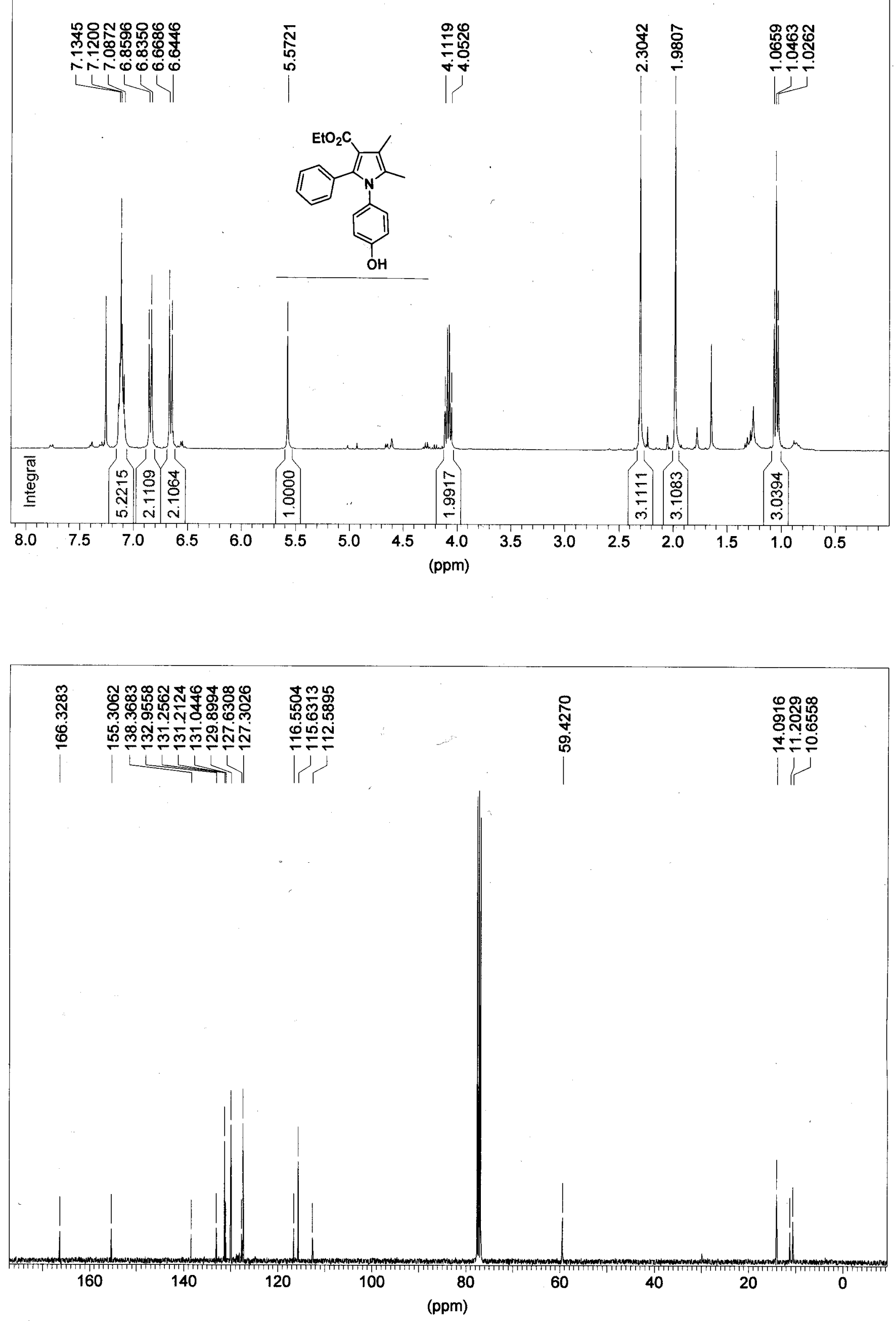

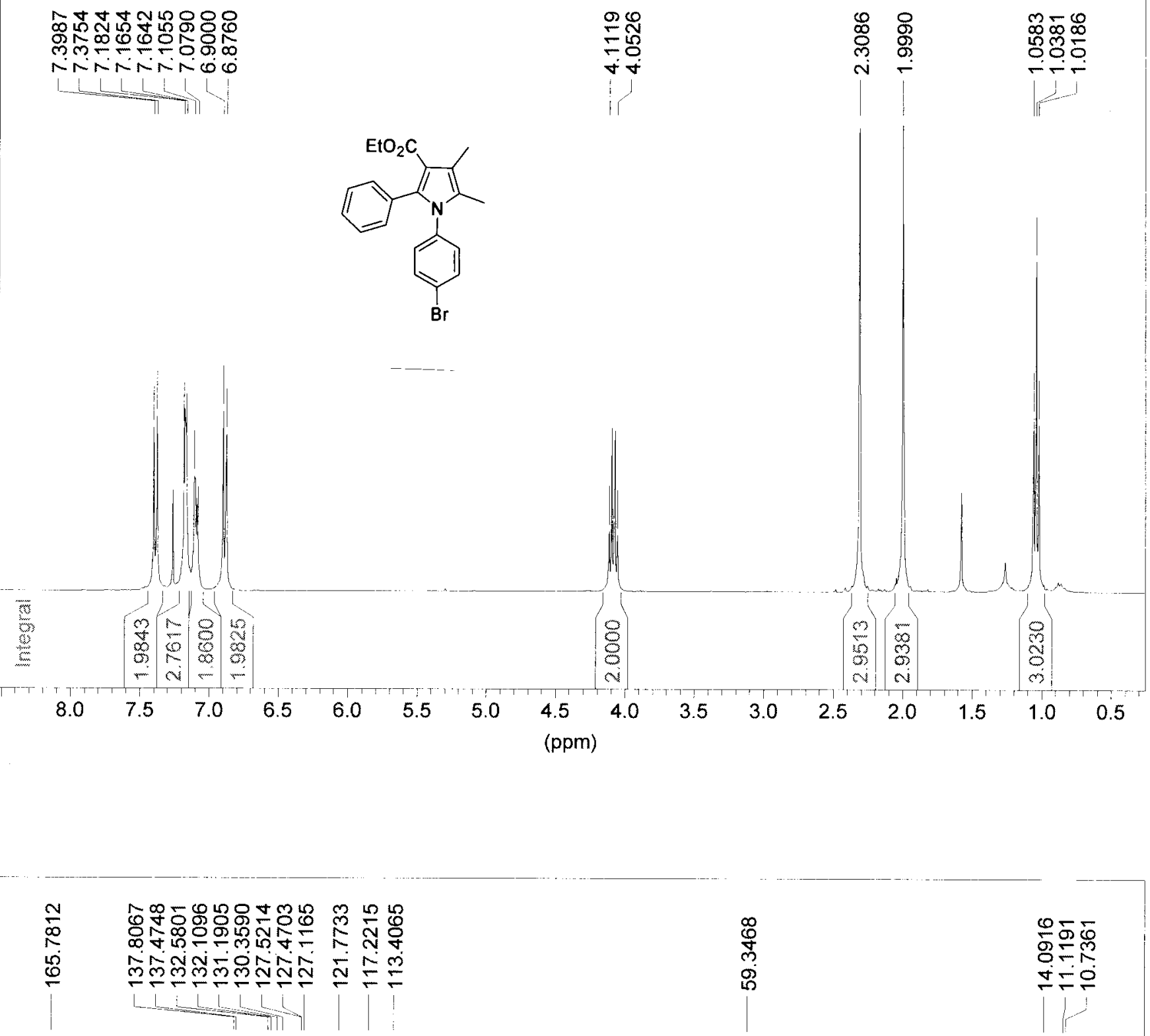

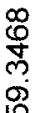
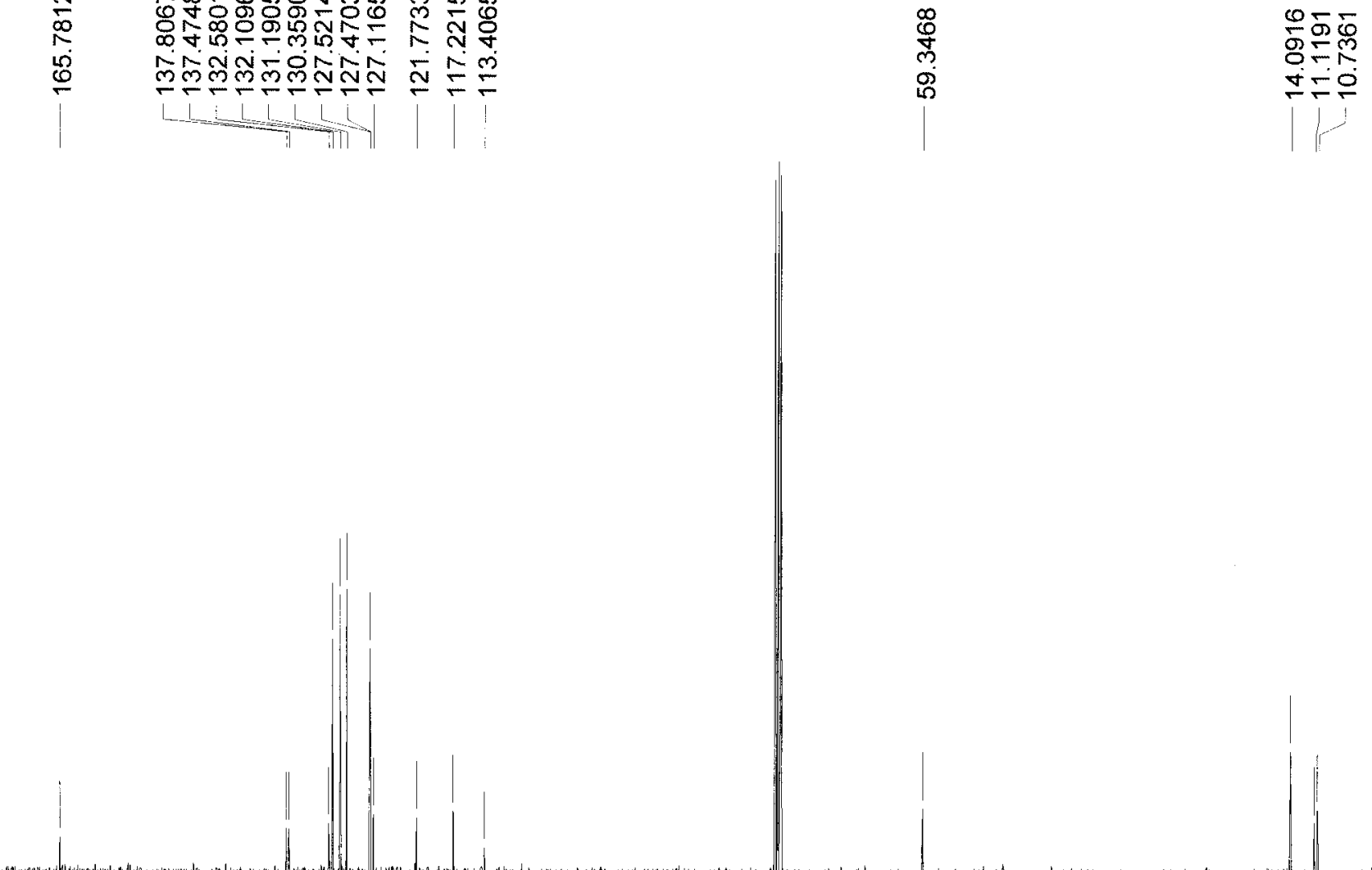

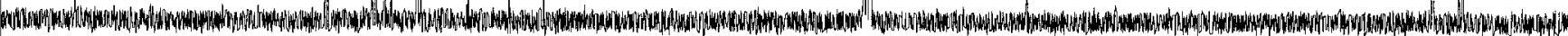



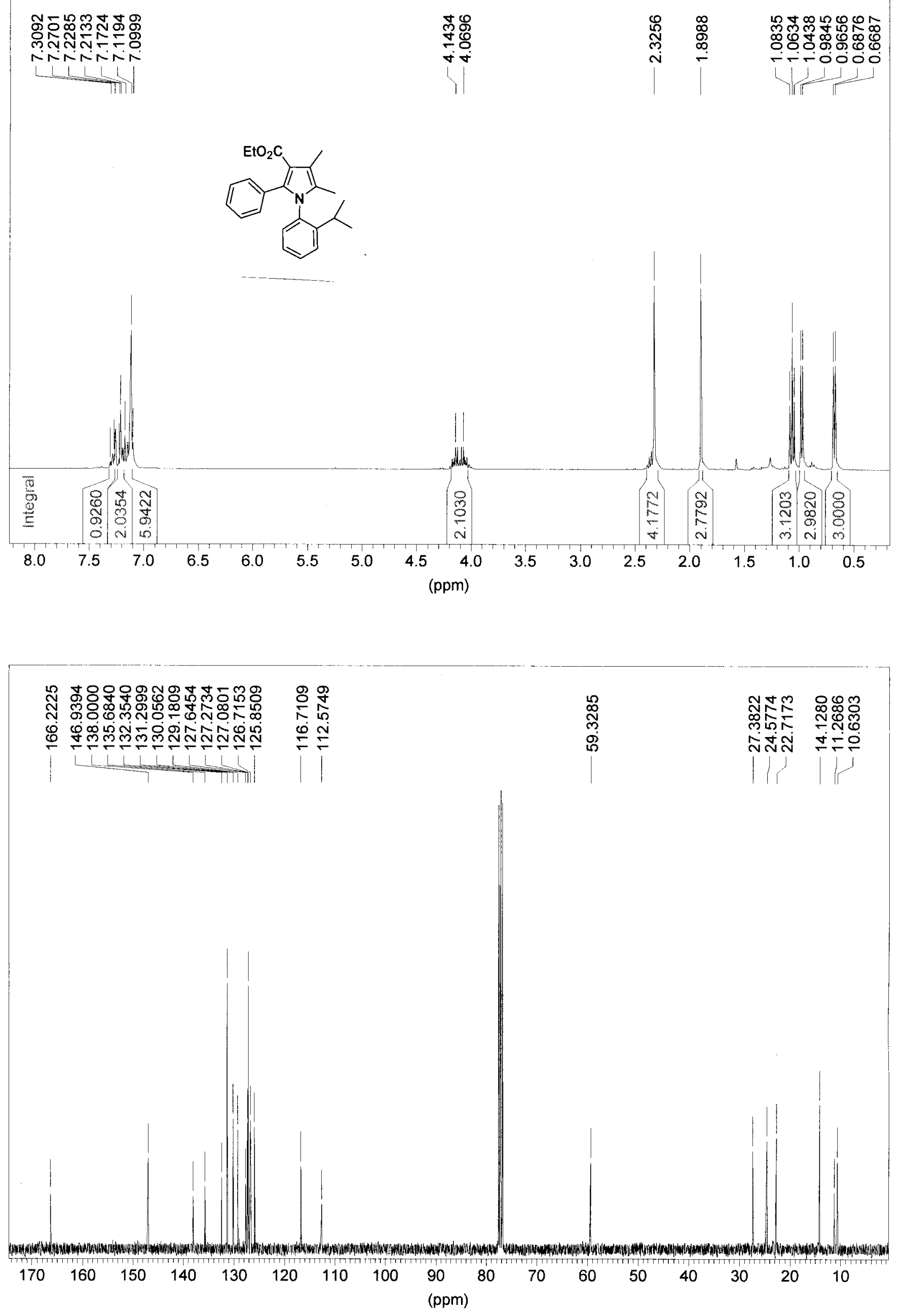


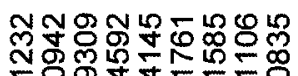

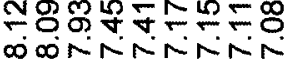

LLLLLLLL!

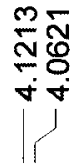

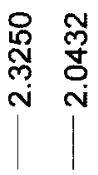

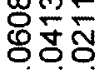

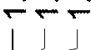<smiles>CCOC(=O)c1c(C)c(C)n(-c2cccc([N+](=O)[O-])c2)c1-c1ccccc1</smiles>

3ah

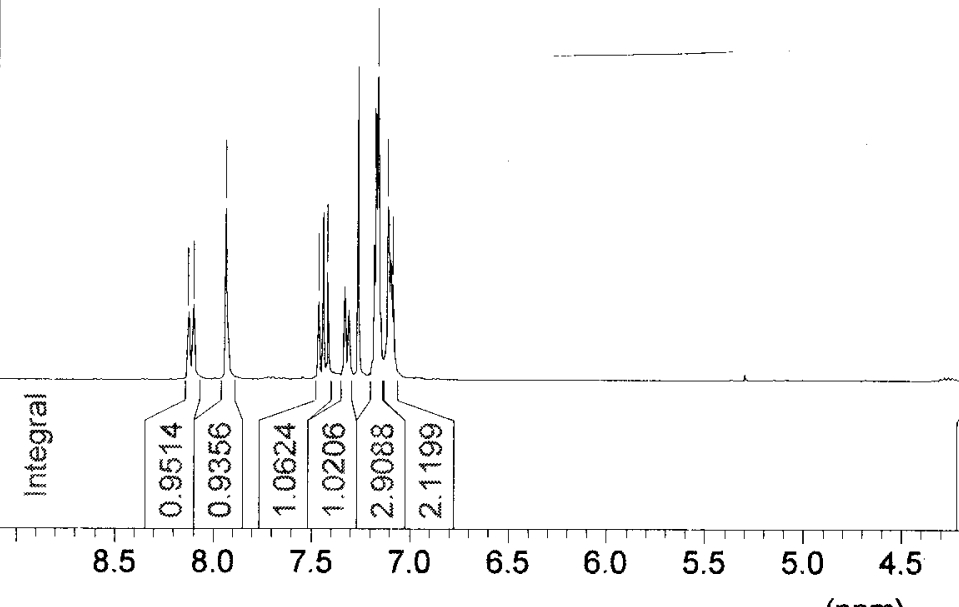

(ppm)
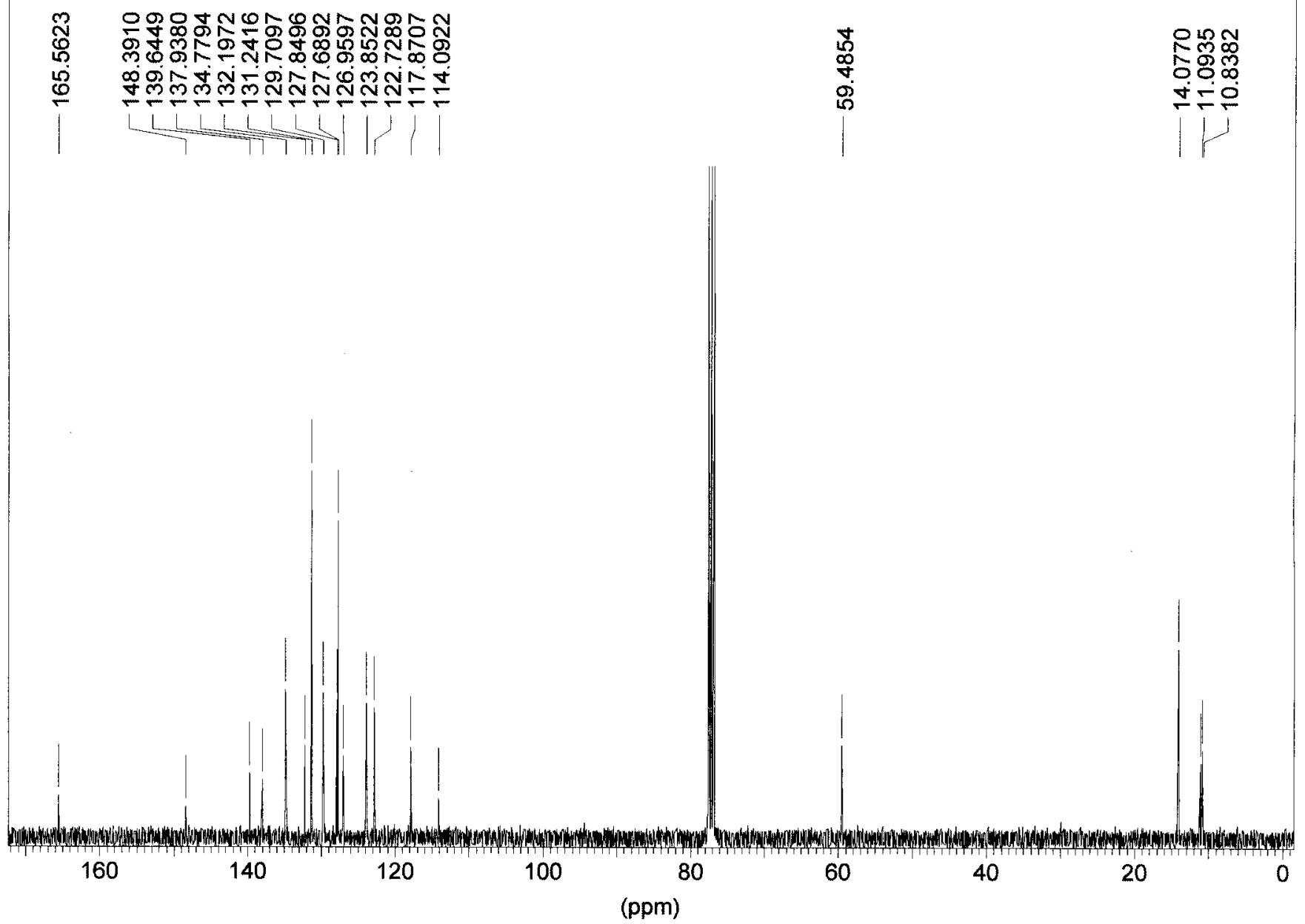

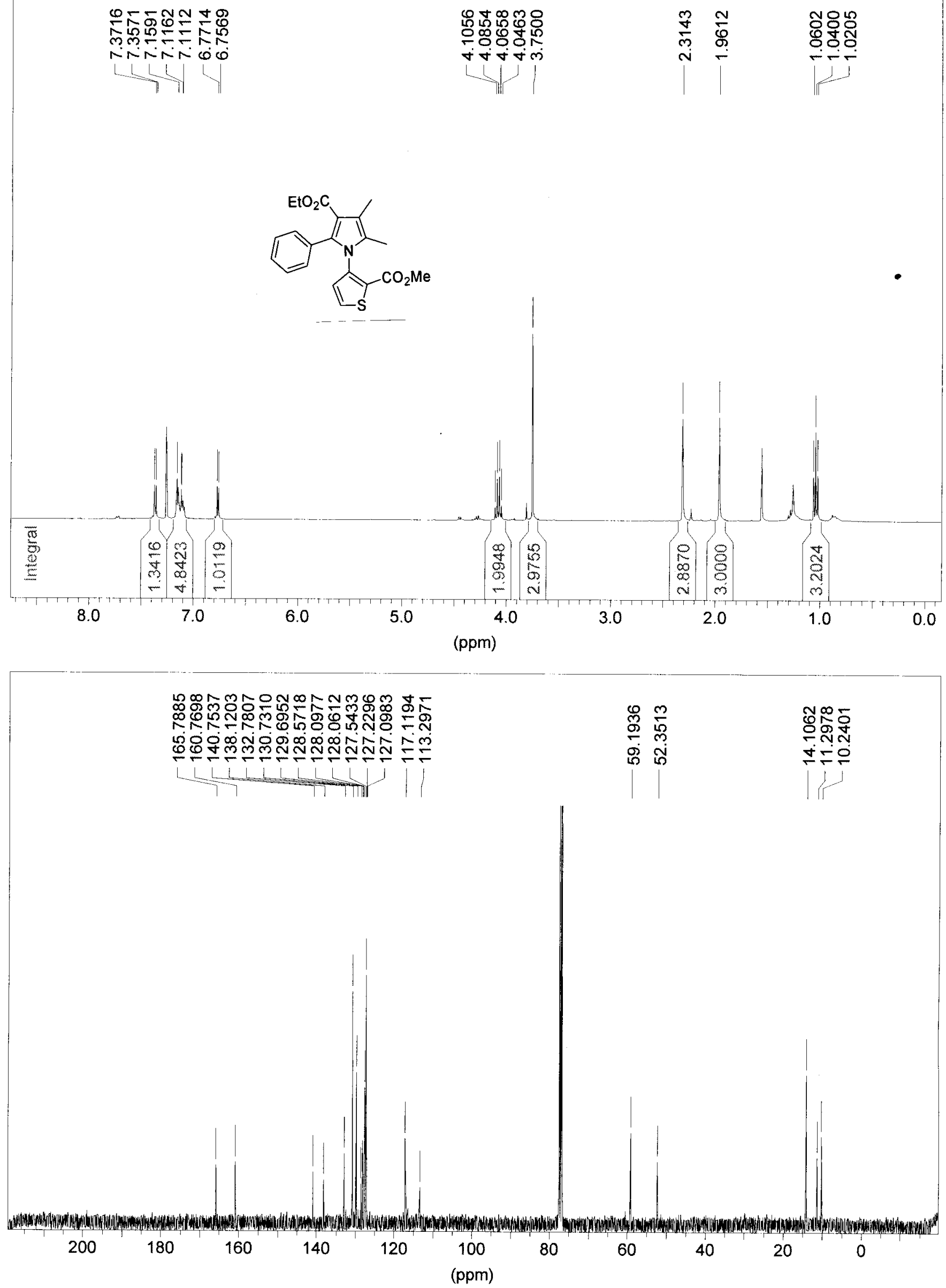

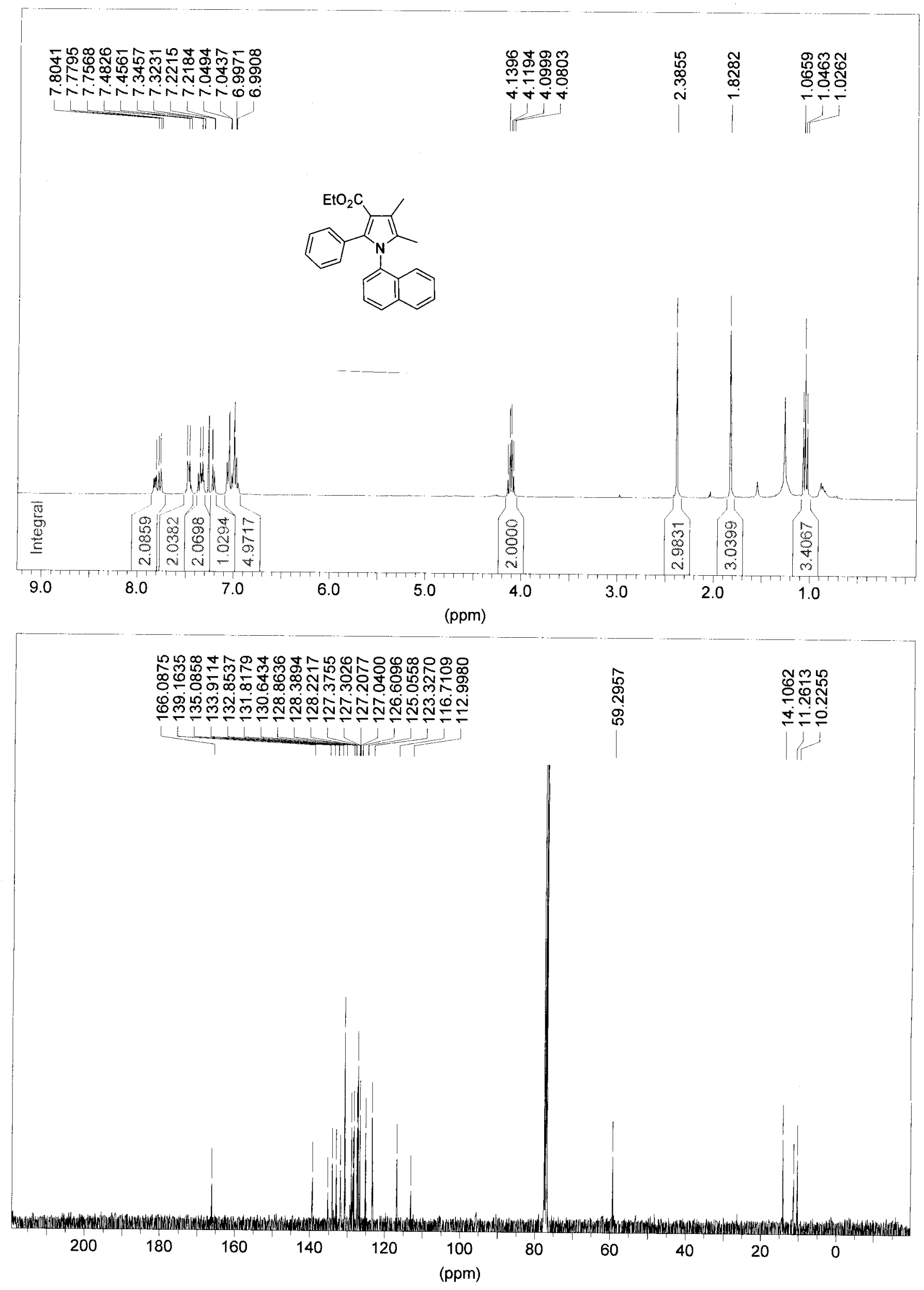


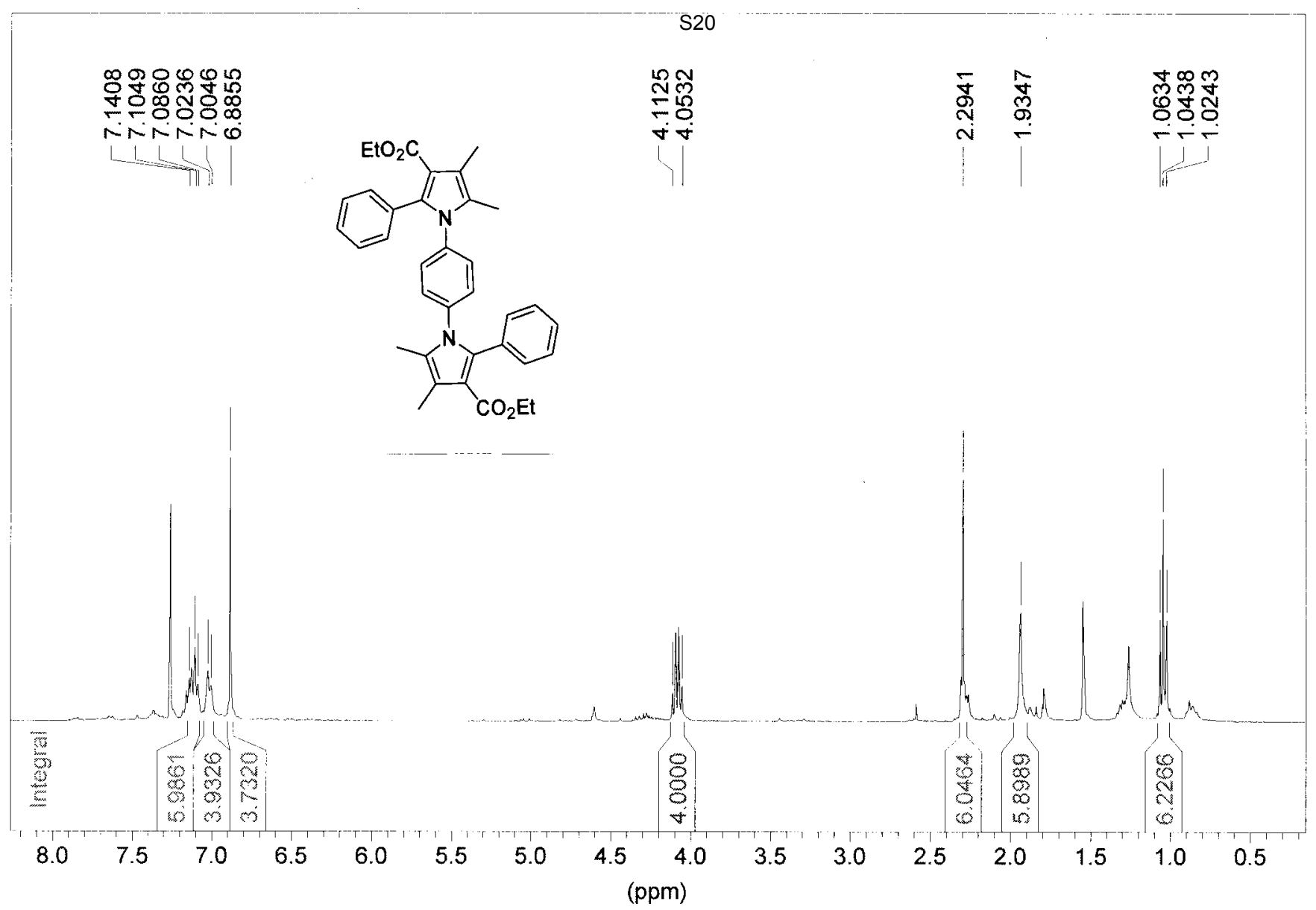

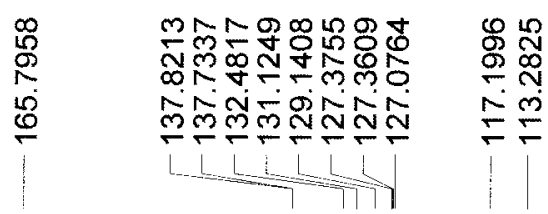

孪

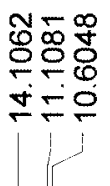

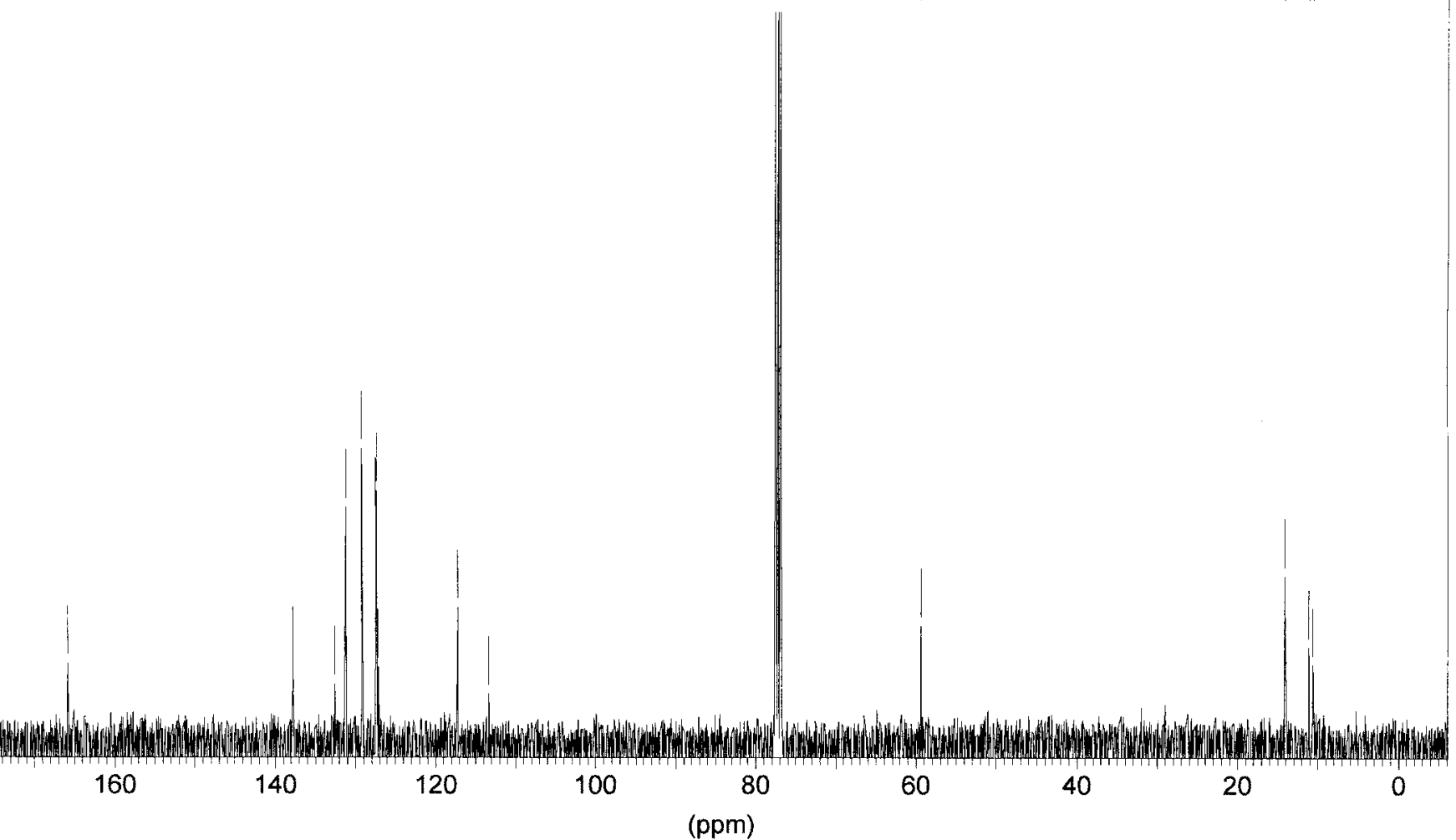




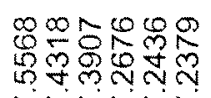

$\stackrel{\infty}{N} \frac{1}{\sim}$

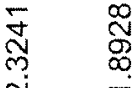

NNNN

!

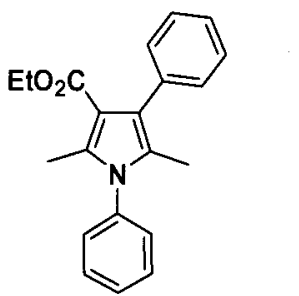

ba

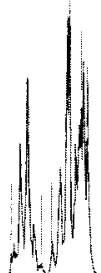

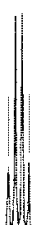

$8 s$

7.5

7.0

6.5

6.0

5.5

5.0

(ppm)

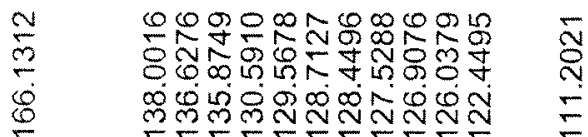

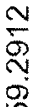

స్유뮤

ษNi?

$\sim$

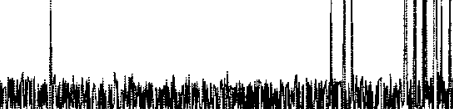




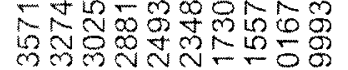

NNMNNMNO

a 1.

\section{$\stackrel{200}{0}$}

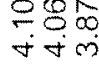

$\underset{\infty}{\infty} \quad \infty$

$\stackrel{\square}{0}$

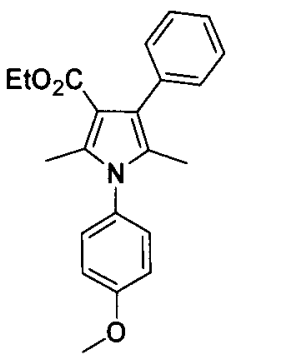

$3 b b$
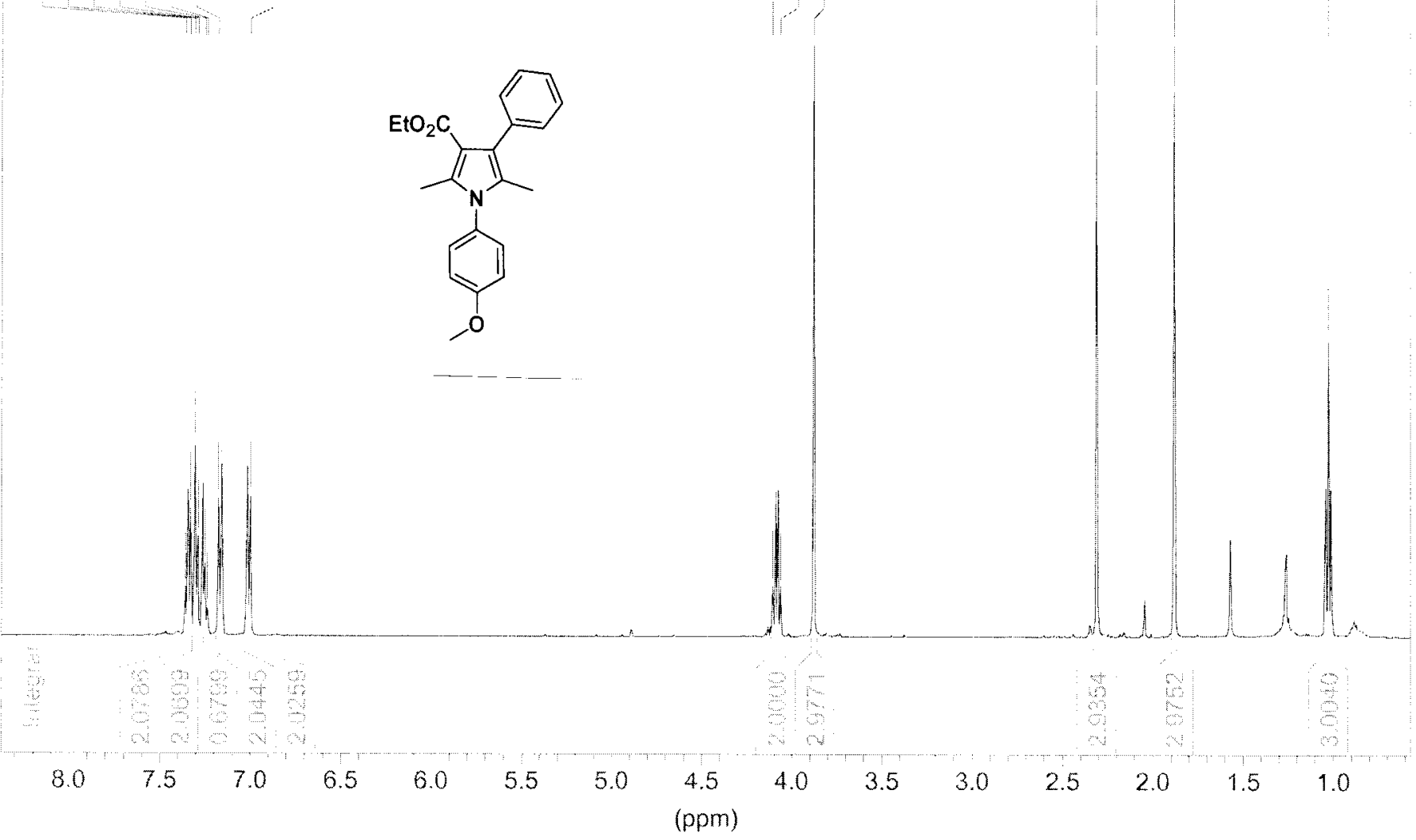

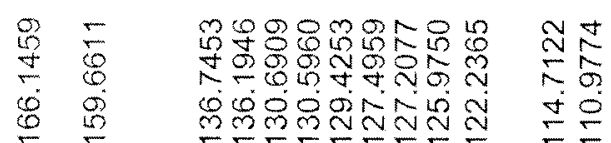

垫早

吕

융용

INE

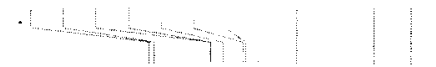

. 


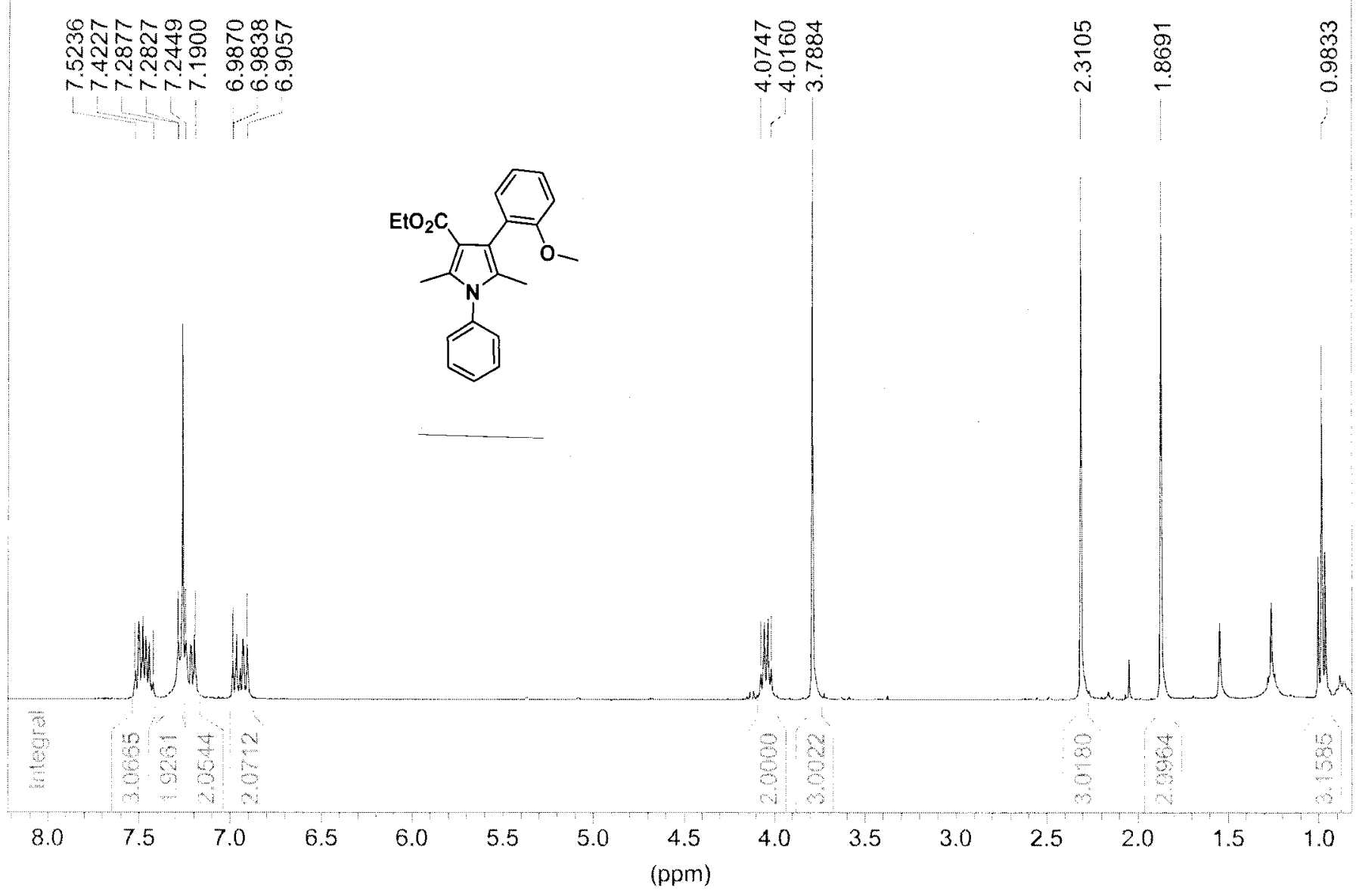

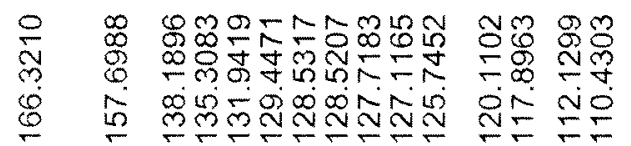

옹

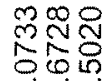

贾品

$\dot{I} \underline{-}$
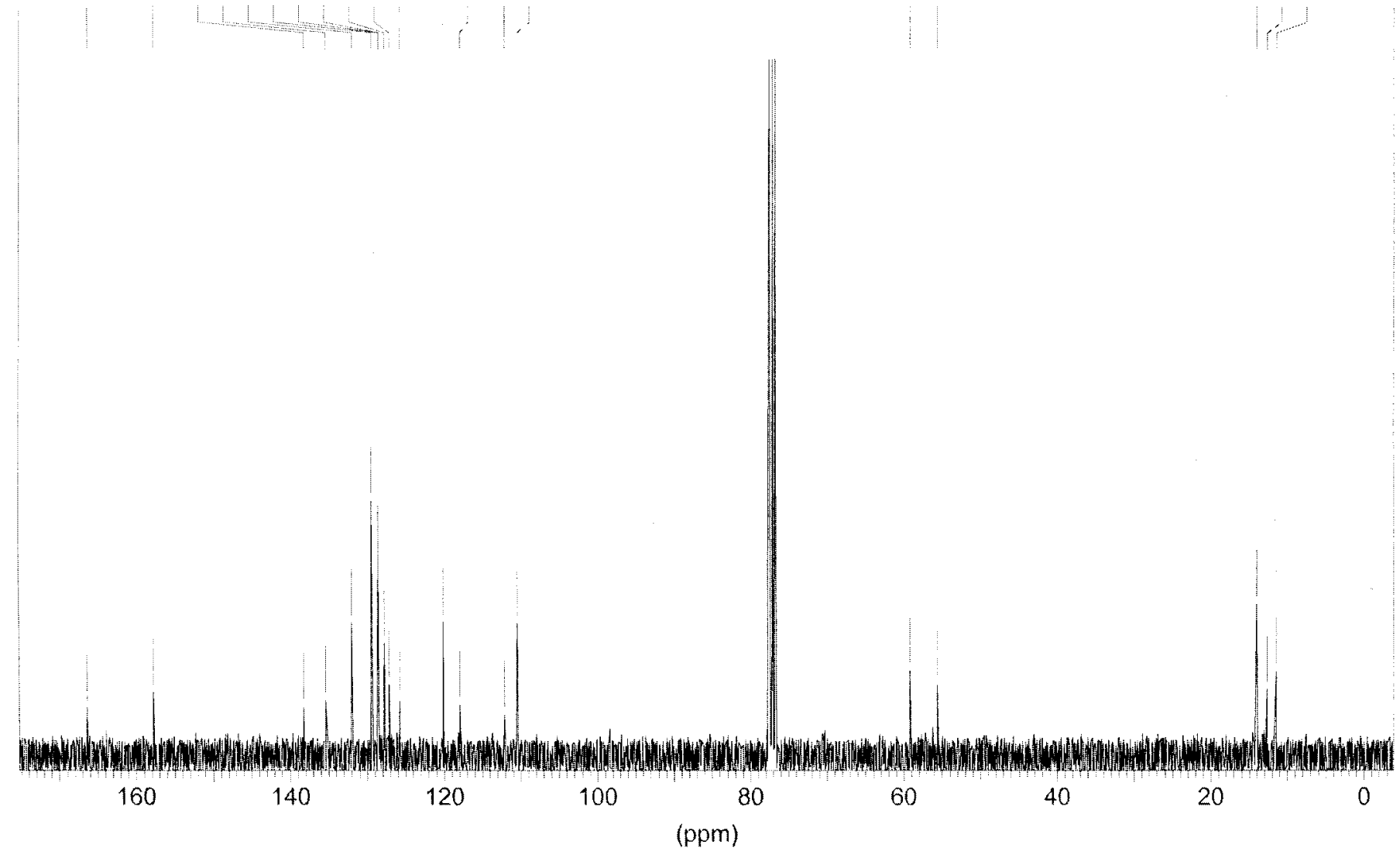

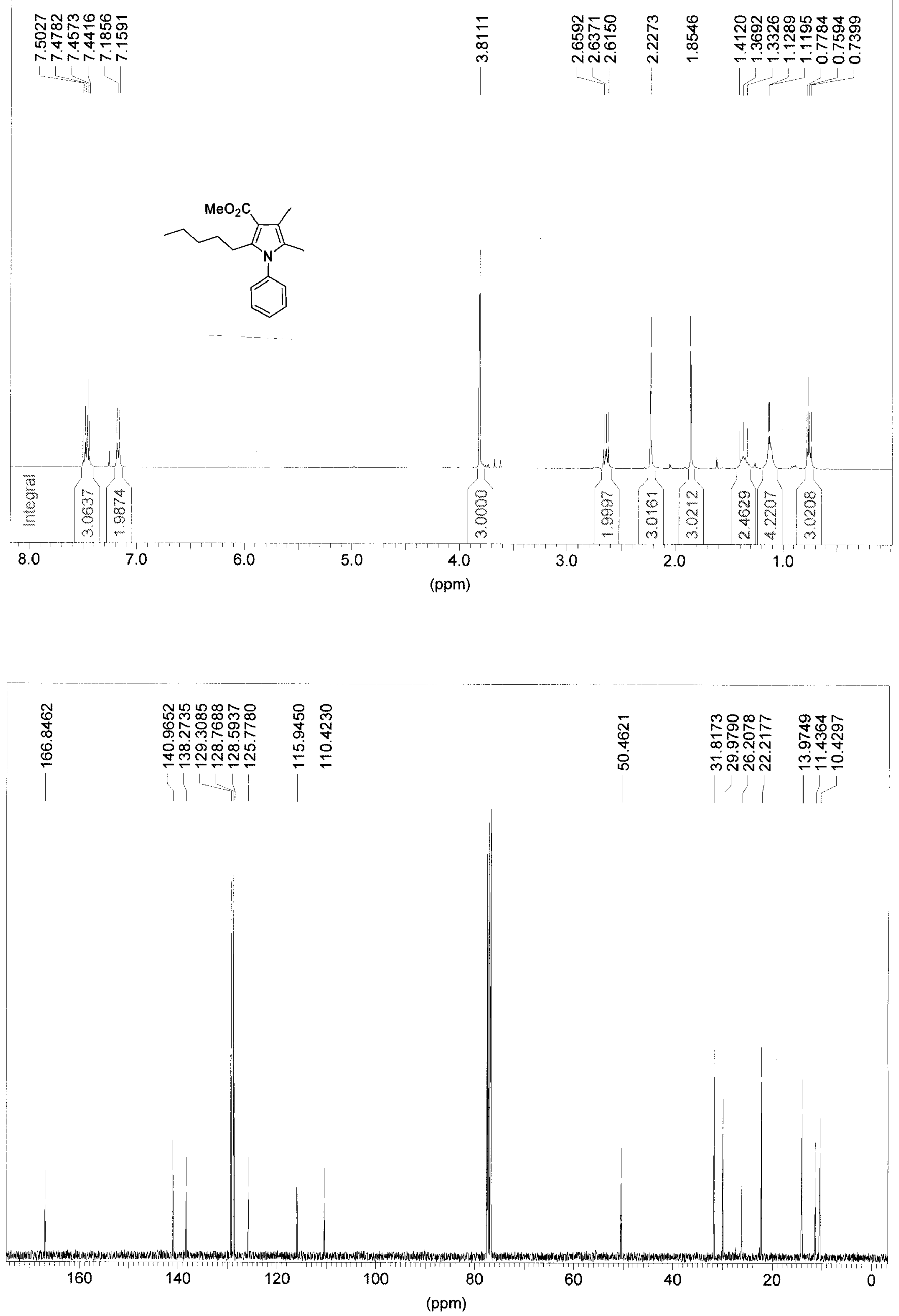

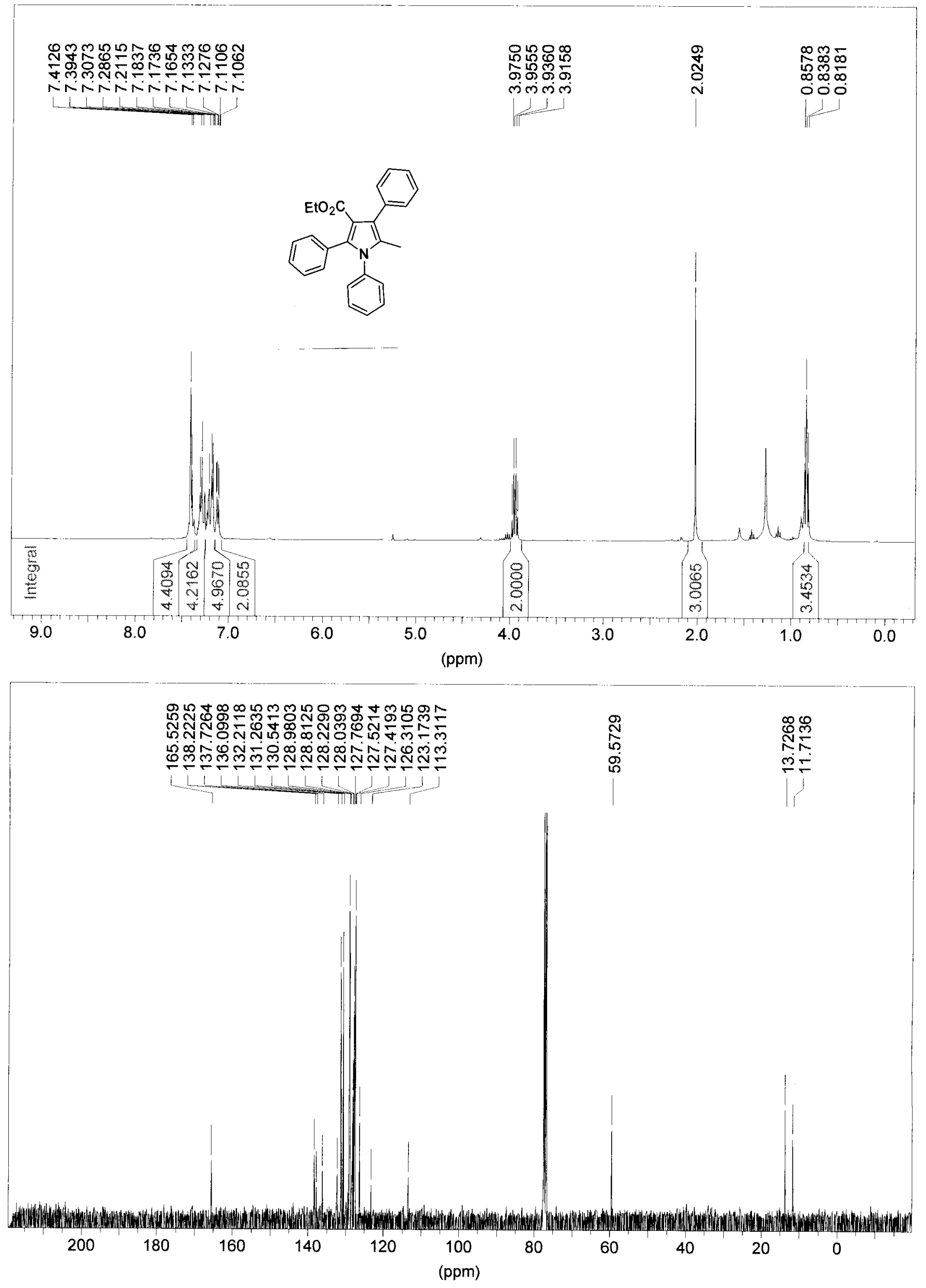

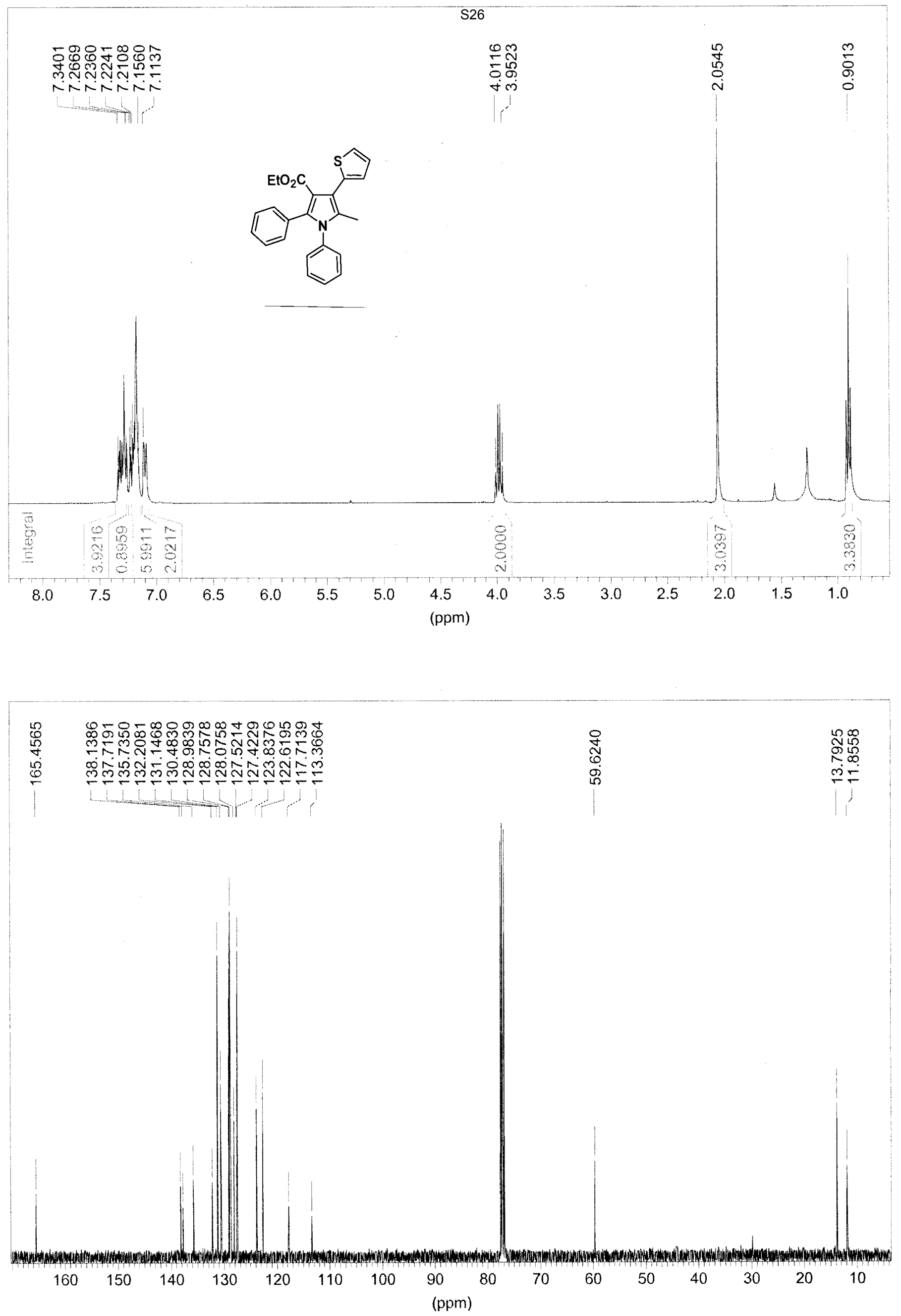


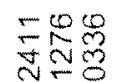

Nis

$\mathbb{1} 0$
$\infty$
$=0$
+10

$m \infty$
$\operatorname{mon}^{\infty}$

옷ำ

vo

OLR

(

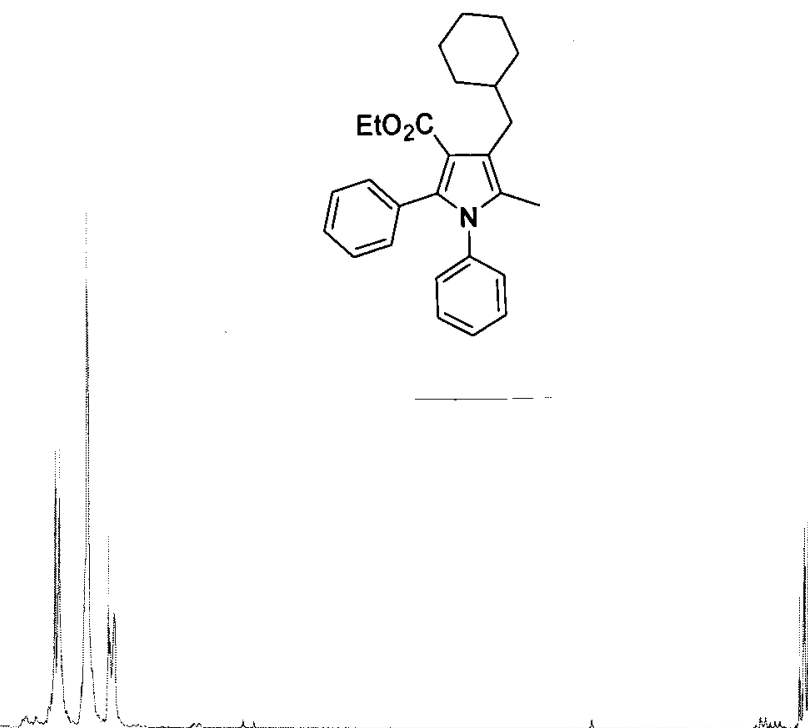

3ga

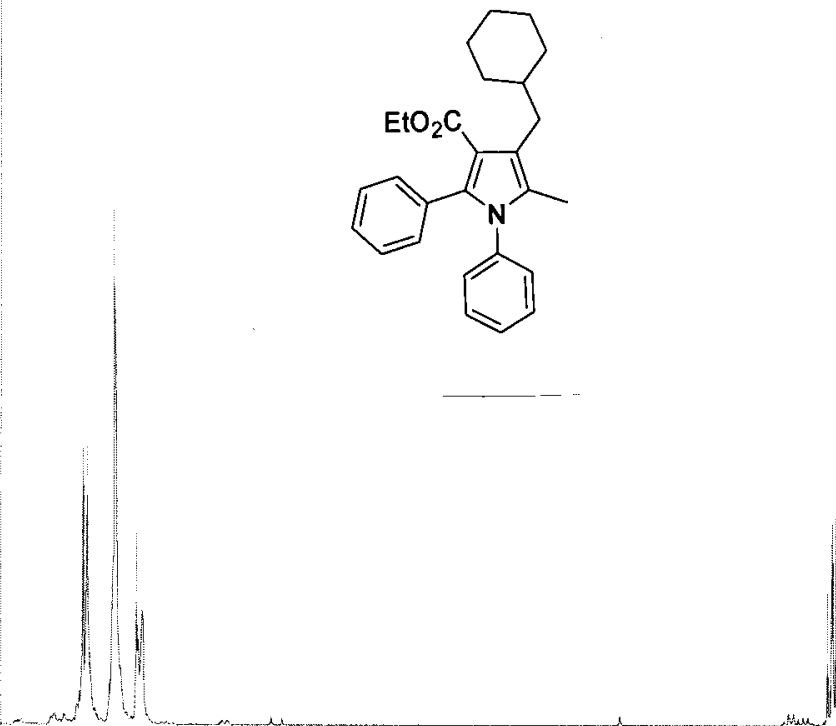

a
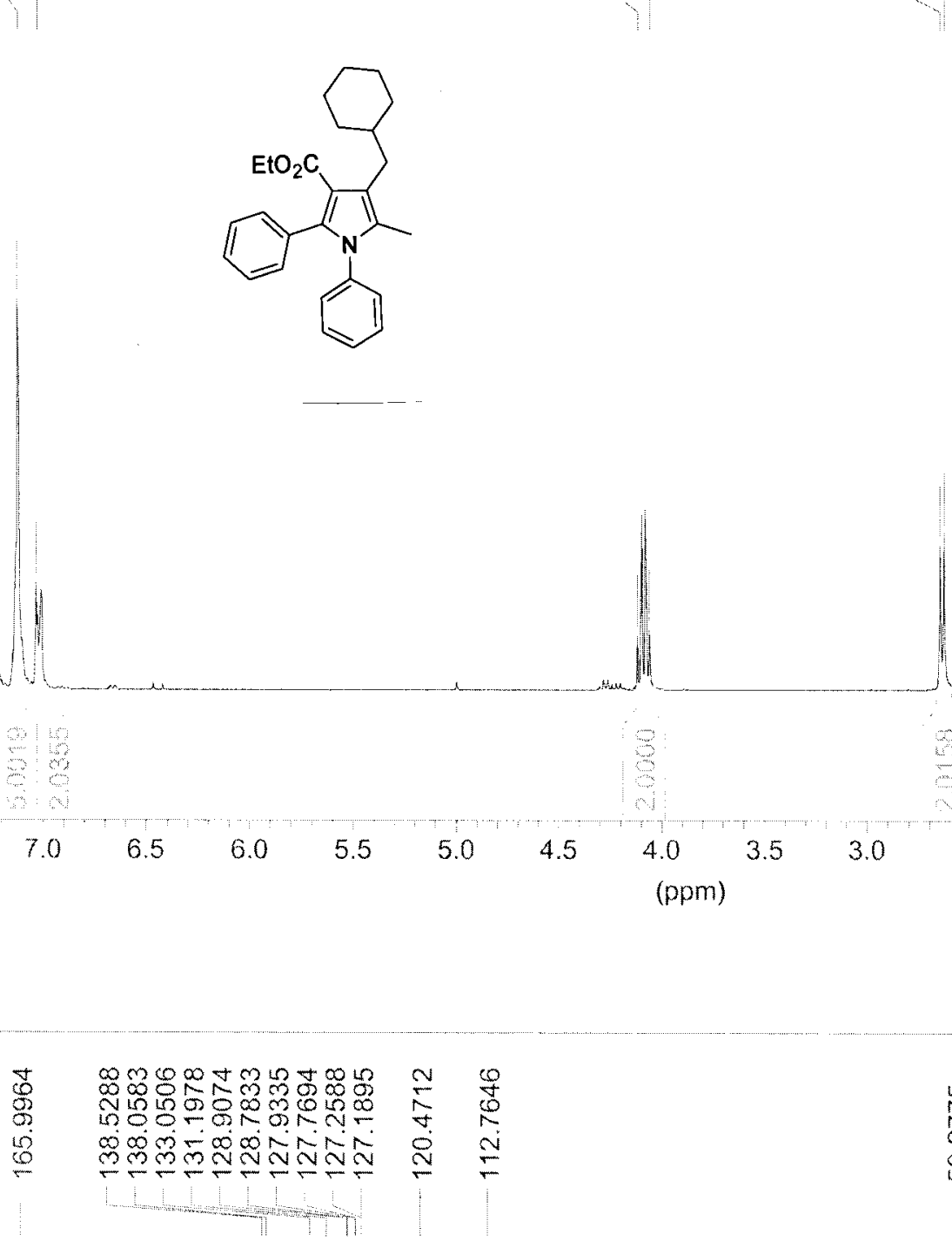

$\stackrel{n}{N}$

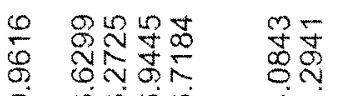

in

ल लmळब्र

$\pm$

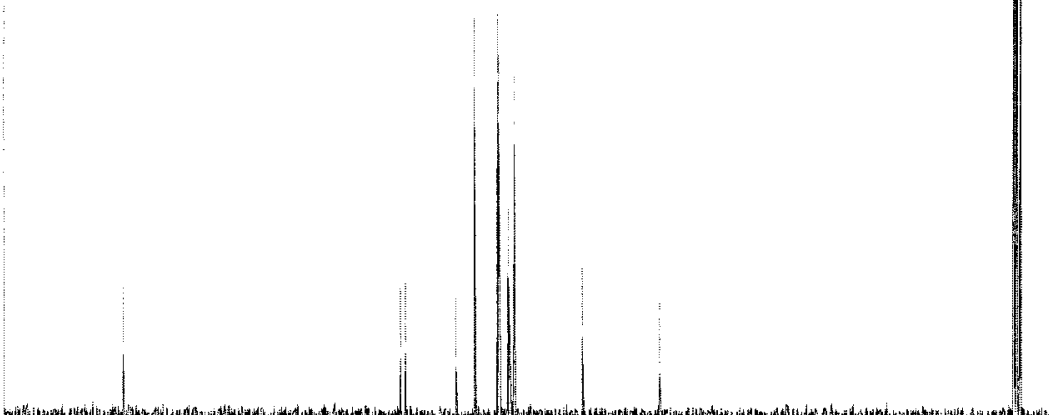

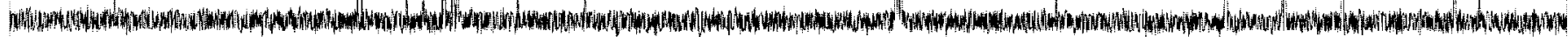

130

120

110

100

90

80

70

60

50

40

$30 \quad 20$

(ppm) 


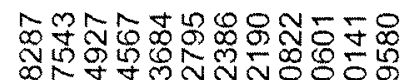

MNNNNNANRO

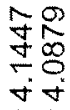

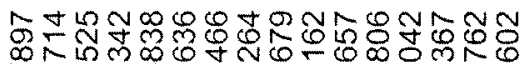

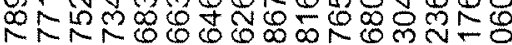

งกัง
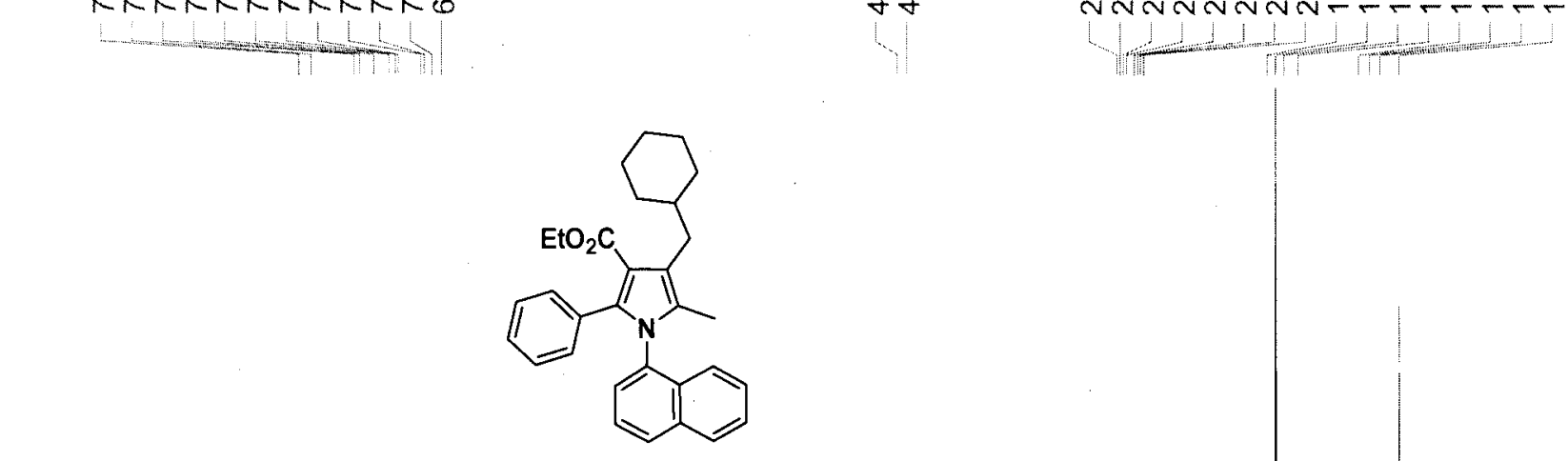

gj
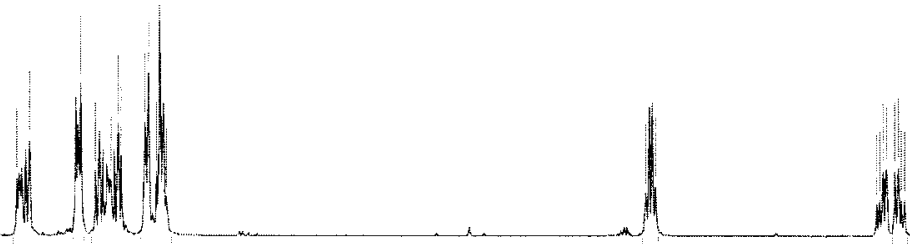

$\sqrt{n}$
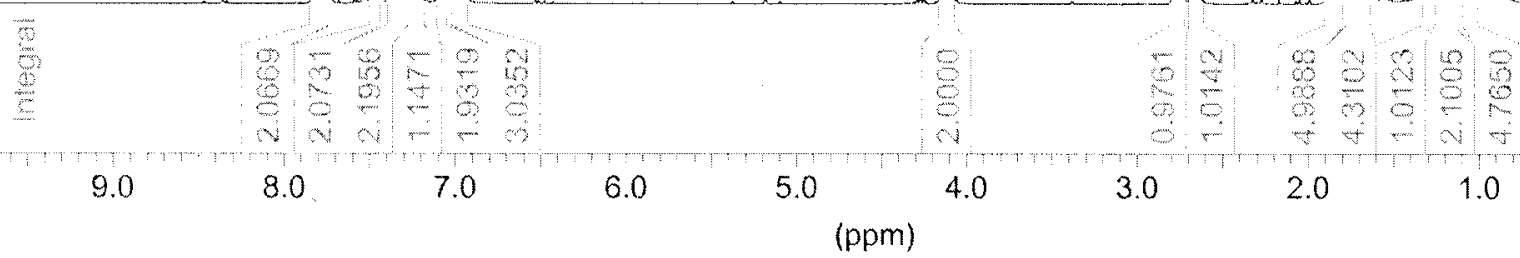

30

1.0

0.0

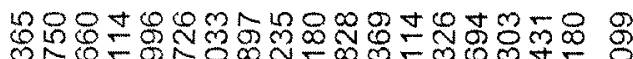

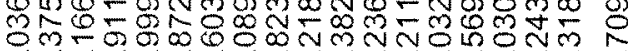

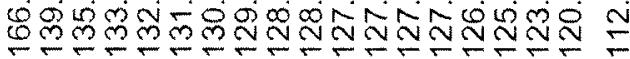

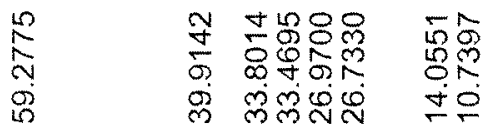

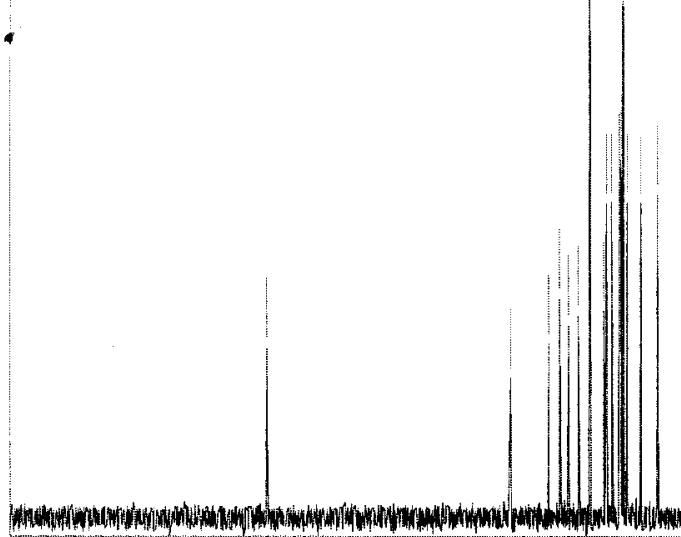

120 

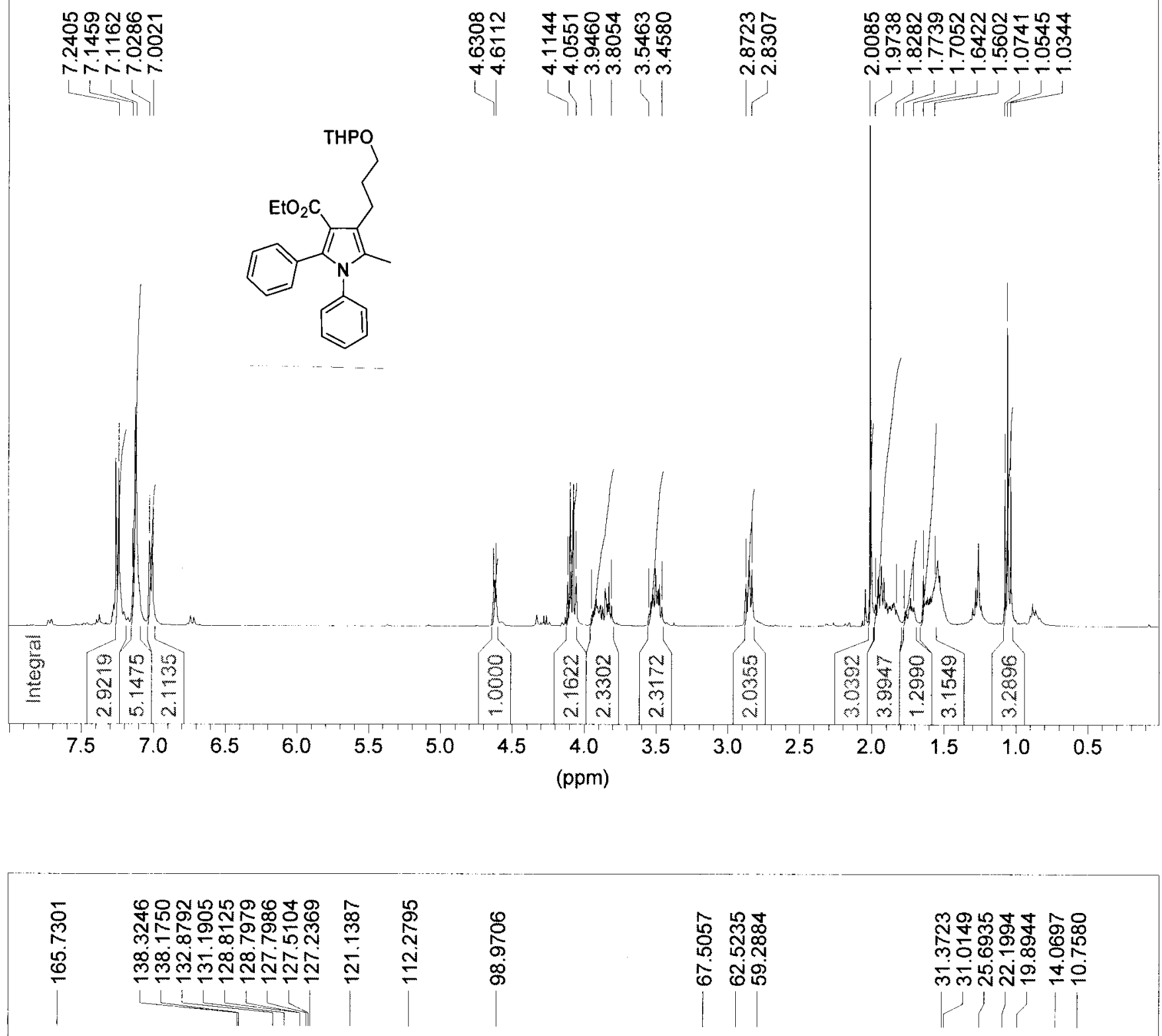

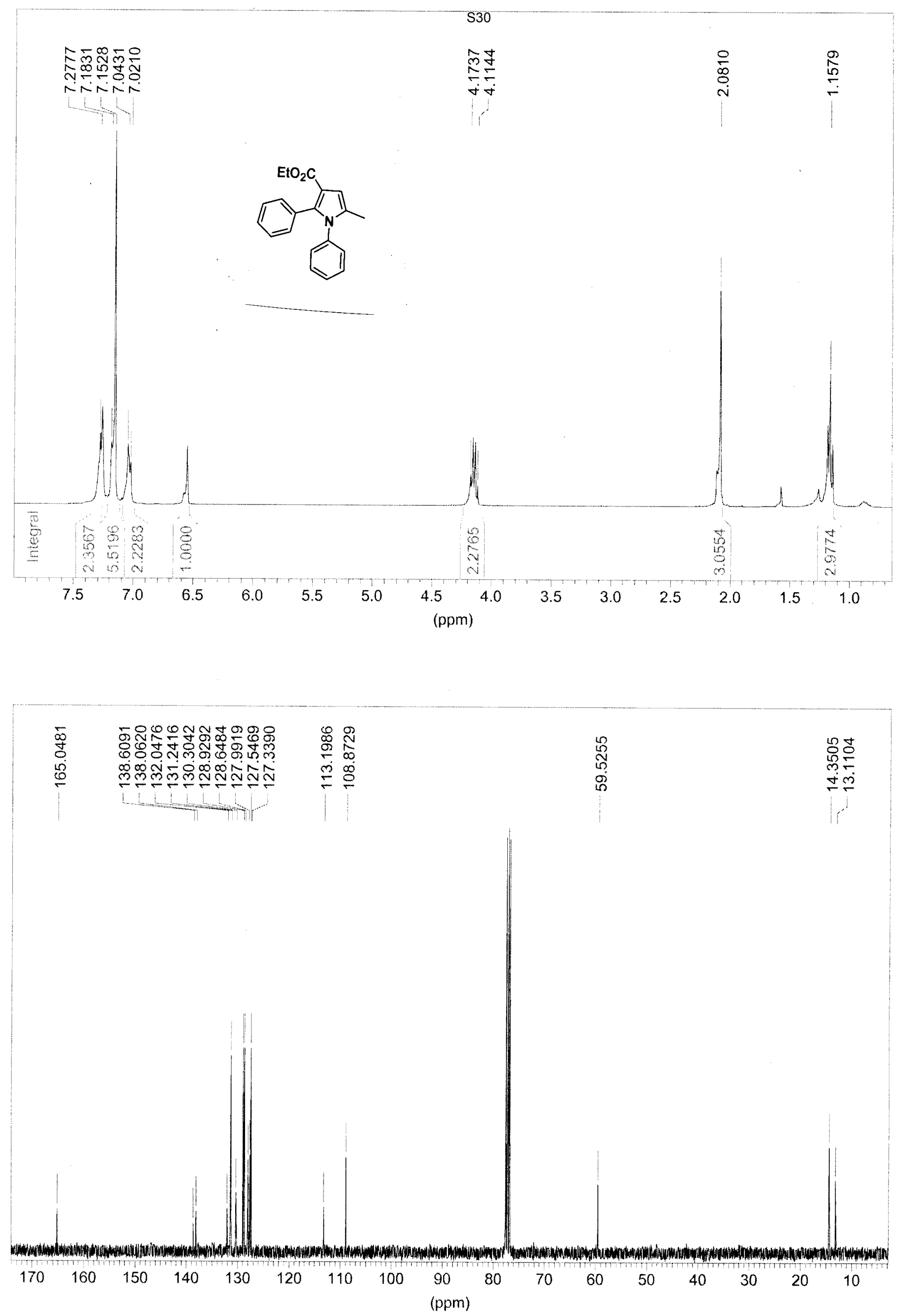


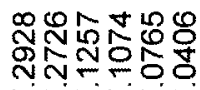

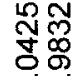

NANAN

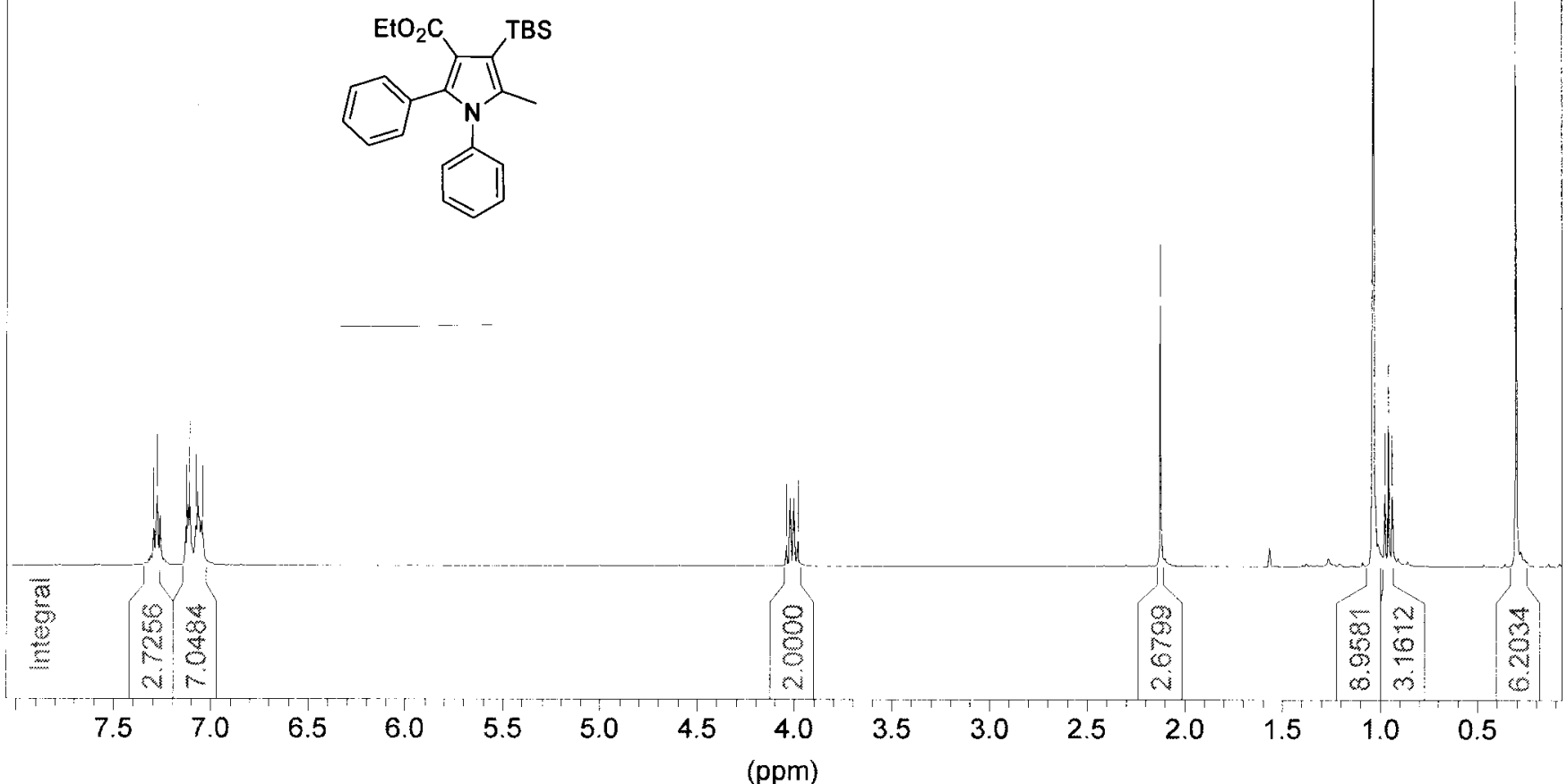

ja

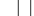

- 00

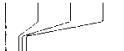

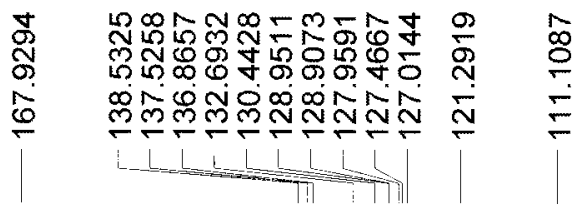

8
80
8
8
8

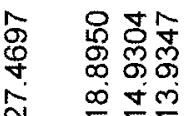

음
ஸे
in

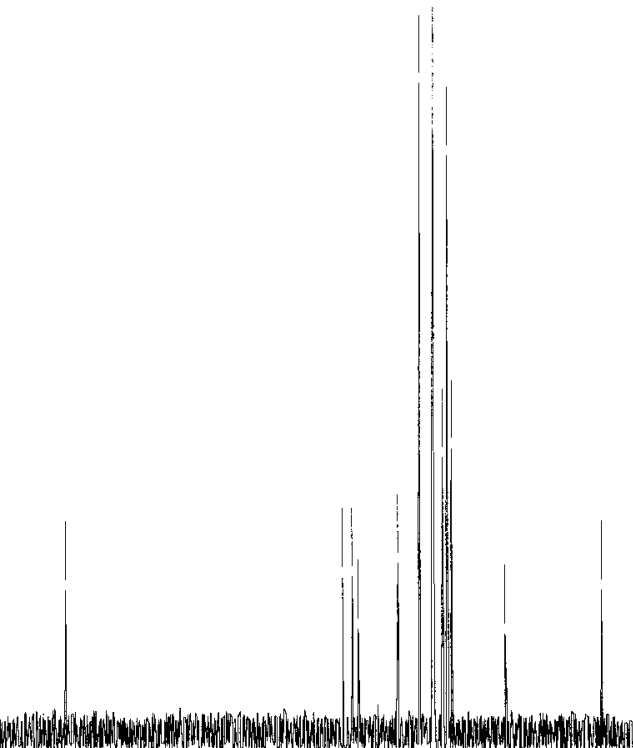

(ppm) 

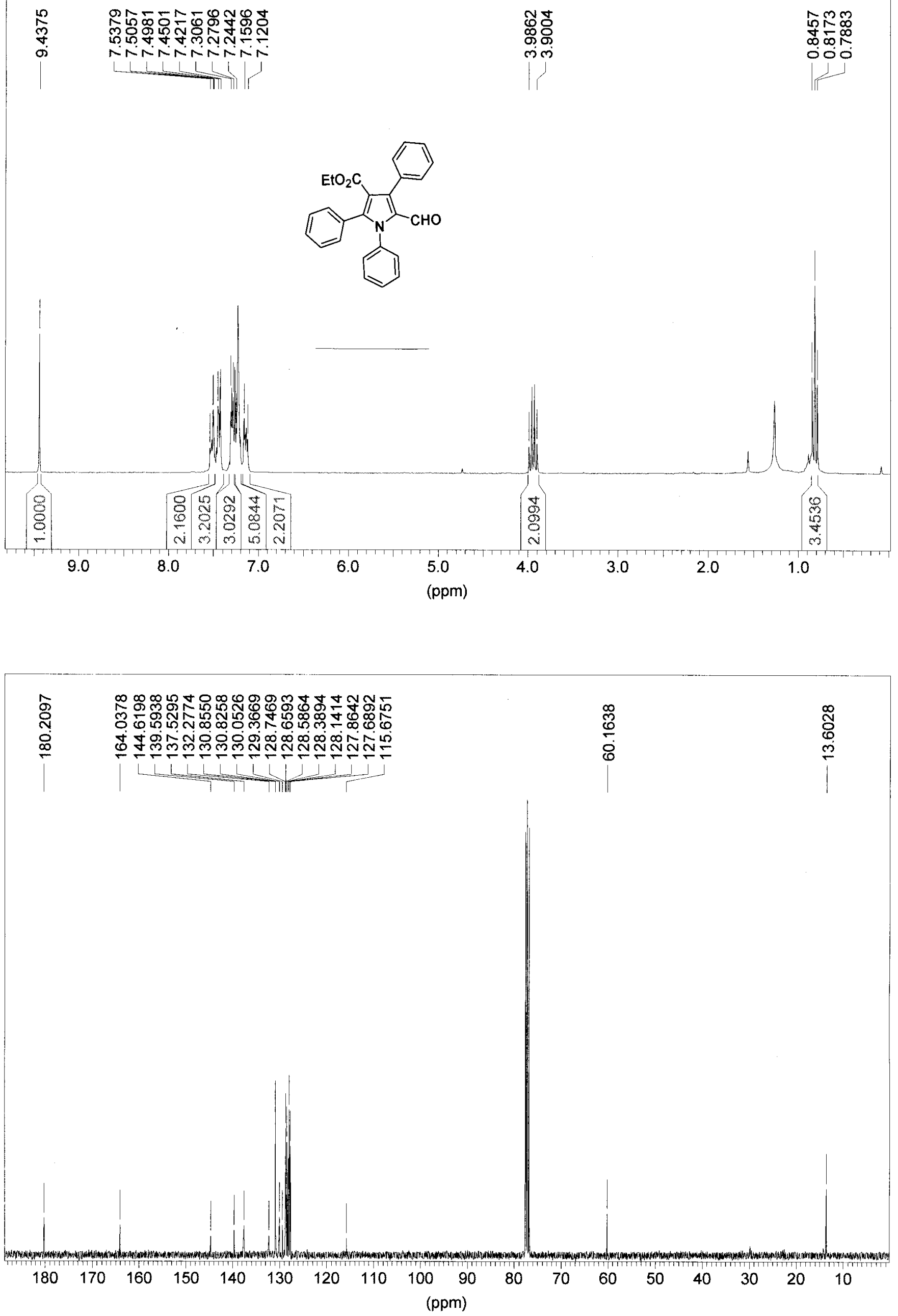

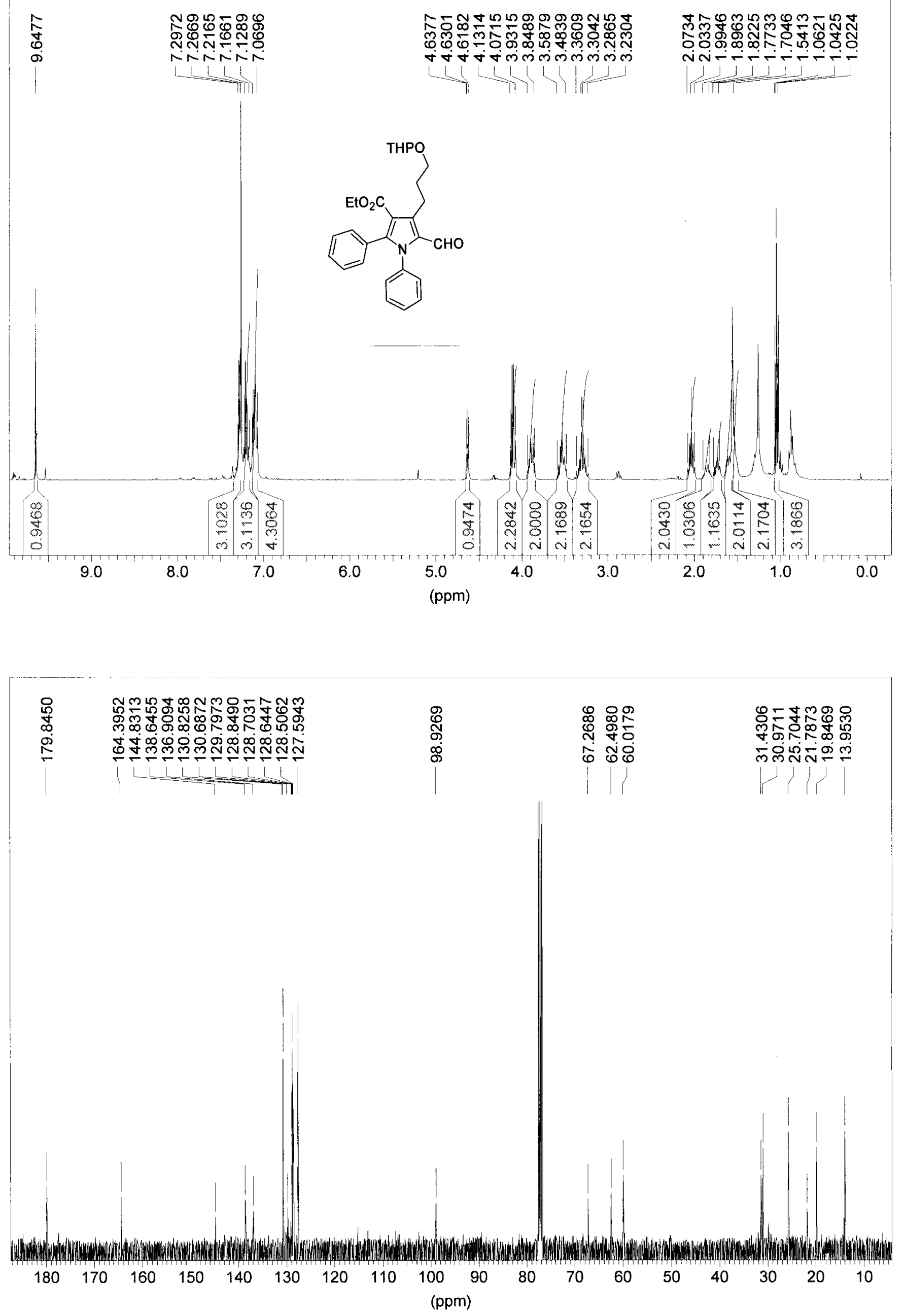

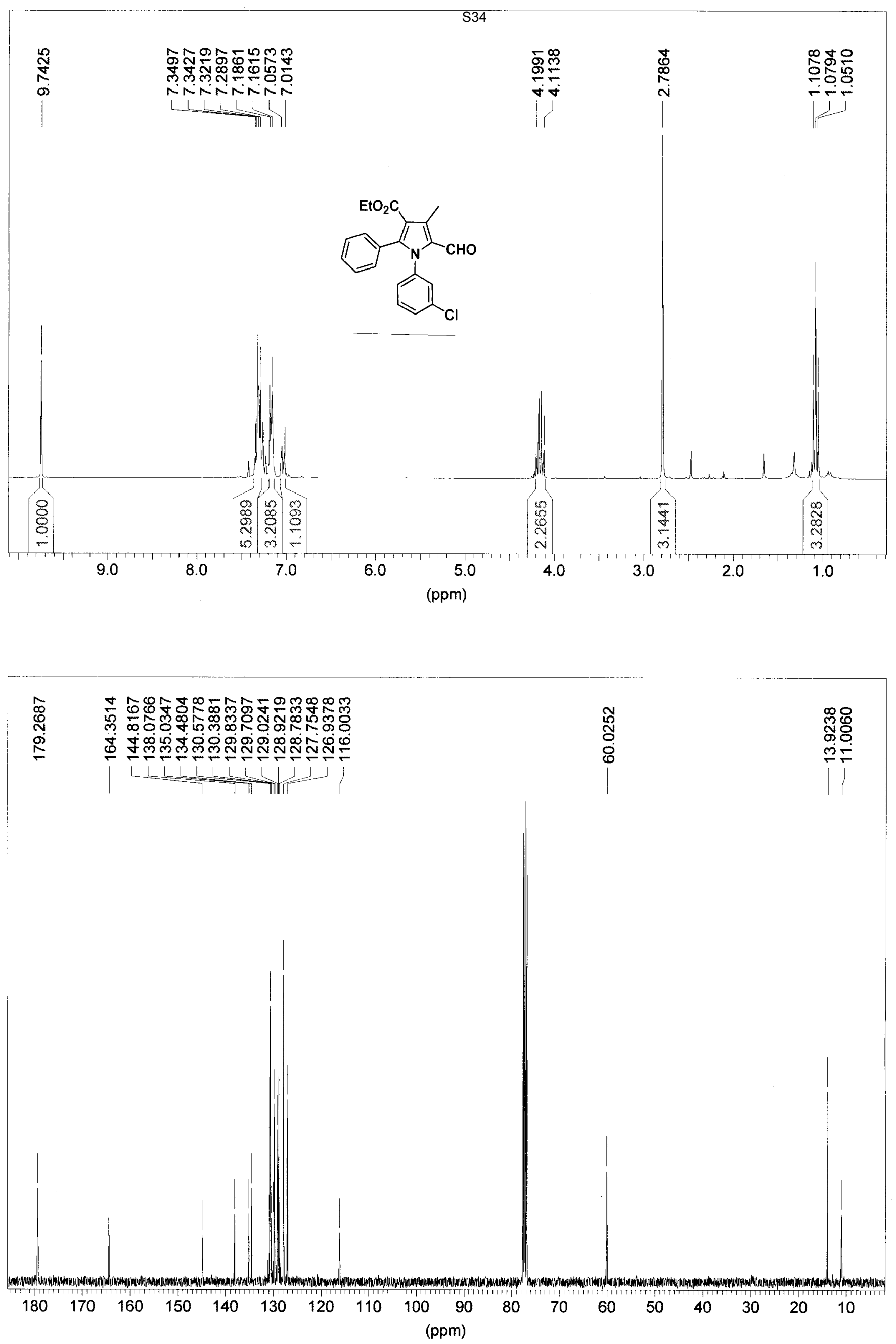
母

母

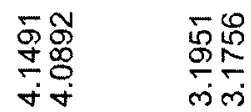

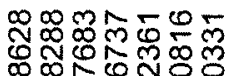

कीजिए।
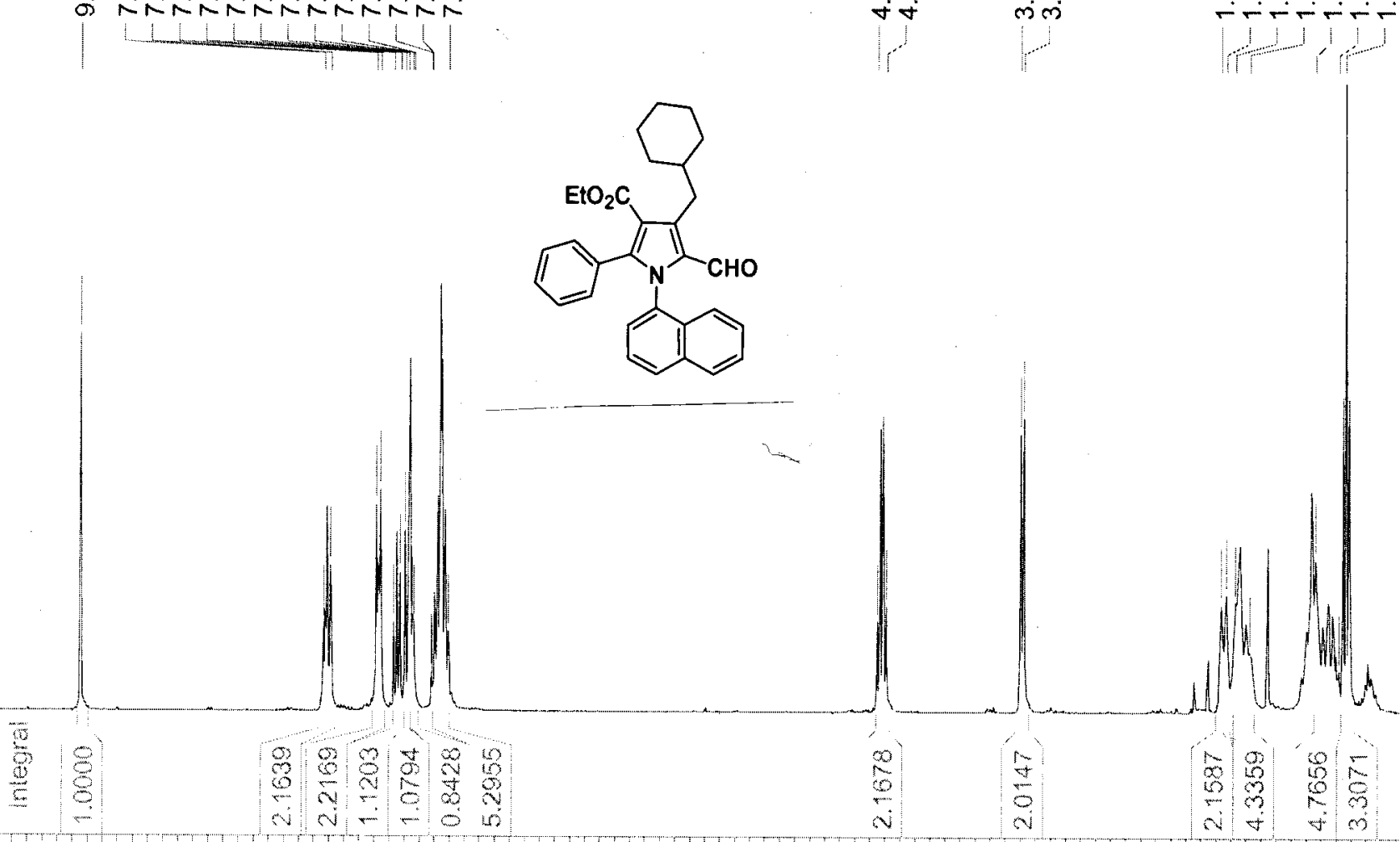

9.0

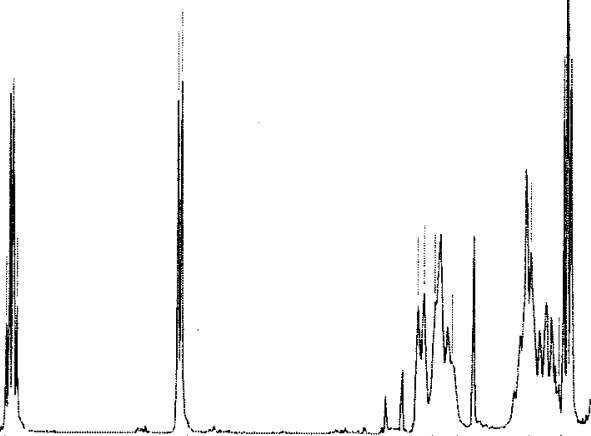

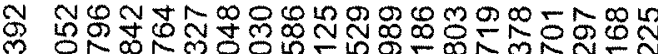

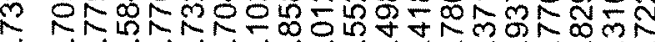

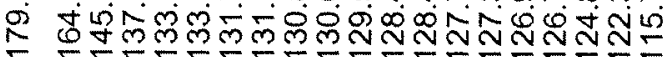

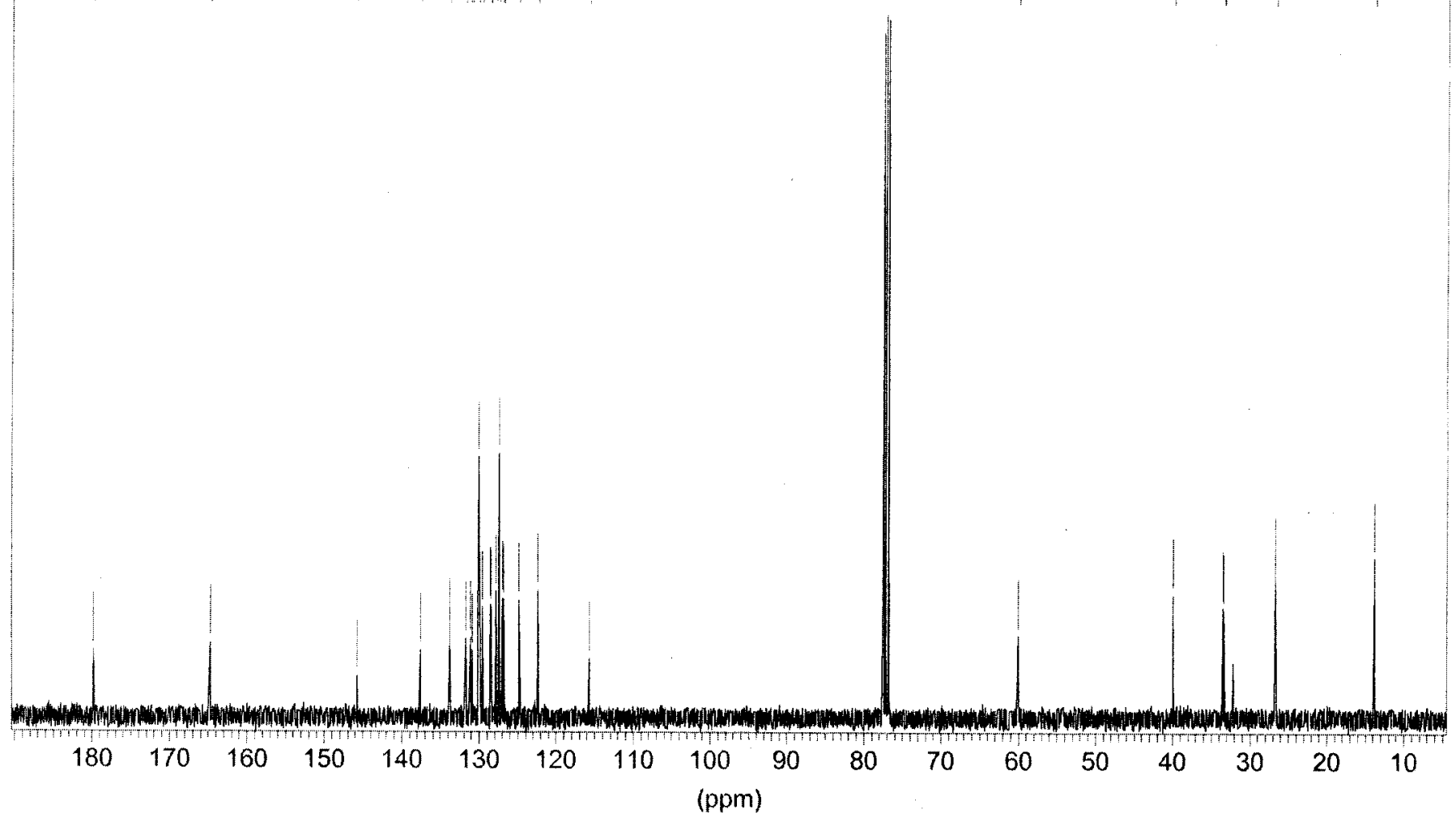

